\title{
FABRICATION OF 3D MILI-SCALE CHANNELS FOR HEMODYNAMIC STUDIES
}

\author{
E. Doutel ${ }^{1}$, J. Carneiro ${ }^{1}$, M. S. N. Oliveira ${ }^{2}$, J.B.L.M. $\operatorname{Campos}^{1}$, J. M. Miranda $^{1}{ }^{(*)}$ \\ ${ }^{1}$ Centro de Estudos de Fenómenos de Transporte, Departamento de Engenharia Química, \\ Faculdade de Engenharia da Universidade do Porto, Rua Dr. Roberto Frias, 4200-465 Porto \\ Portugal \\ ${ }^{2}$ Department of Mechanical and Aerospace Engineering, University of Strathclyde, Glasgow \\ G1 1XJ, UK
}

\begin{abstract}
3D mili-scale channel representing simplified anatomical models of blood vessels were constructed in polidimethylsiloxane (PDMS). The objective was to obtain a sequential method to fabricate transparent PDMS models from a mold produced by rapid prototyping. For this purpose, two types of casting methods were compared, a known lost-wax casting method and a casting method using sucrose. The channels fabricated by both casting methods were analysed by Optical Microscopy, Scanning Electron Microscopy (SEM) and Energy Dispersive X-Ray Spectroscopy (EDS). The lost-wax method is not ideal since the channels become contaminated during the removal process. The models produced with the lost-sucrose casting method exhibit much better optical characteristics. These models are transparent with no visible contamination, since the removing process is done by dissolution at room temperature rather than melting. They allow for good optical access for flow visualization and measurement of the velocity field by micro-particle image velocimetry ( $\mu \mathrm{PIV}$ ). The channels fabricated by the lost-sucrose casting method were shown to be suitable for future hemodynamic studies using optical techniques.
\end{abstract}

Keywords: 3D channels, blood vessels, lost-sucrose casting, $\mu$ PIV

*Corresponding author: Fax: +351 225081449, e-mail: jmiranda@fe.up.pt 


\section{INTRODUCTION}

Cardiovascular diseases are presently one of the leading causes of death in humans ${ }^{1}$. From clinical practice, it is known that specific sites in the human circulatory system are particularly sensitive to the development of cardiovascular diseases ${ }^{2}$. Local hemodynamics is crucial to understand the development and the complications derived from medical conditions like atherosclerosis ${ }^{3,4}$, aneurysms ${ }^{5-8}$ and stenoses ${ }^{9-11}$. Some medical interventions, such as stent implantation and bypasses, can disturb regular hemodynamics ${ }^{12-14}$ and their impact on fluid flow must be understood.

Fluid dynamics studies have been used to advance the understanding of cardiovascular diseases. For example, Shahcheraghi et al. ${ }^{15}$ studied numerically three-dimensional and pulsatile blood flow in a human aortic arch and its three major branches, suggesting that the preferential development of lesions is in regions of extreme wall shear stresses and pressure. These results are in agreement with the numerical data of Kobayashi and Pereira ${ }^{16}$. Barakat and Cheng ${ }^{17}$ presented a numerical simulation of fluid disturbance induced by intravascular stents. They demonstrated the existence of a region of flow separation immediately downstream the implemented stent.

The study of fluid flow of the vascular system requires a combination of numerical and in vivo and in vitro experimental studies. In vivo studies are only possible in very limited situations due to practical and ethical reasons. Numerical studies always require experimental validation due to the importance of turbulence, non-newtonian effects and, for microvessels, the particulate nature of blood. The velocity of blood flow in vitro has been studied using several techniques, including: video microscopy and image analysis ${ }^{18}$, laser-Doppler anemometry ${ }^{19}$ and particle-based methods such as Particle Tracking Velocimetry (PTV) ${ }^{20}$, Particle Image Velocimetry (PIV) and Micro-Particle Image Velocimetry ( $\mu$ PIV) ${ }^{21,22} . \mu$ PIV was applied to the study of microcirculation ${ }^{21,23,24}$, flows in vessels with less than $250 \mu \mathrm{m}$. Confocal $\mu$ PIV ${ }^{24}$ has become accepted as a reliable method for measuring velocity profiles of biological flows in vessels of small diameter with high spatial resolution. Lima et al. ${ }^{23}$, demonstrated the ability of confocal $\mu$ PIV to measure both homogeneous and nonhomogeneous fluids, using red blood cell suspensions. In vitro studies require the construction of a model of the blood vessel under study. The model must be transparent in the wavelength of the light used and its half thickness must be smaller than the focal distance of the objective used.

Several researchers have been working on patient-specific in vitro techniques that allow experimental studies of blood flow in realistic configurations ${ }^{3,25-27}$. Models of complex geometries are typically constructed by direct rapid prototyping from a $3 \mathrm{D}$ computational representation of the blood vessel ${ }^{26,27}$ or by casting in a mold produced by rapid prototyping 3

There are several studies concerning the use of different sacrificial template materials for the fabrication of in vitro models including wax ${ }^{28-30}$, cerrolow (metallic alloy with a low temperature melting point- 320 to $352 \mathrm{~K}$ ) ${ }^{31-33}$, isomalt (sugar based material - 418 to $423 \mathrm{~K}$ melting point) ${ }^{31}$, gelatine, chocolate and plaster powder ${ }^{34}$. Sucrose ${ }^{35}$ has been used as a sacrificial template material to fabricate 3D networks of cylindrical microchannels. The most commonly reported materials are wax and cerrolow, and in these cases the sacrificial mold is removed from the final mold by melting the material at high temperatures.

The major limitation of wax is that it is a very fragile material and so small diameter channels (less than $2 \mathrm{~mm}$ ) cannot be produced ${ }^{29,31}$. While most of the experimental work in this field has been done with vessel structures larger than $5 \mathrm{~mm}$ and microvessels smaller than $300 \mu \mathrm{m}$, there are very few studies on vessels with diameters in the range between $300 \mu \mathrm{m}$ and $5 \mathrm{~mm}$ and fabrication techniques for this intermediate range would be very useful. Cerrolow has no size limitation, but is well known to leave residues in the vessels wall after melting ${ }^{31,32}$ which is not acceptable for quantitative measurement when optical access is required.

In this work, a multi-stage approach was developed to create and replicate simplified 3D models of arteries in the millimeter-scale range, with or without stenosis, using melted sucrose as casting material. The main difference relatively to other common casting methods and materials is that the removal process in which the sacrificial mold is destroyed is carried out by dissolving the sucrose in water at room temperature, rather than melting, which allows to produce transparent non-contaminated PDMS channels suitable for flow visualizations and optical flow measurements. 
These studies were performed to ensure that the fabricated channels are suitable for hemodynamics studies, i. e., are able to mimic realistically the geometry of an artery, are easily replicated and reproducible and exhibit ideal optical properties and no contamination.

The paper is organized as follows. The fabrication process is described and discussed in detail in section 2. In section 3, the PDMS transparent channels are analyzed by Optical Microscopy, Scanning Electron Microscopy (SEM) and Energy Dispersive X-Ray Spectroscopy (EDS). Flow dynamics studies conducted using flow visualization and $\mu$ PIV techniques are presented in section 4. We finalize the paper with concluding remarks in section 5 .

\section{FABRICATION OF IN VITRO MODELS}

An overview of the fabrication process is illustrated in Figure 1. The geometry is created in Computer-aided design (CAD) and a first mold made of an epoxy-resin is then constructed by rapid prototyping by stereolithography. Afterwards, a negative version of the mold is fabricated in non-transparent silicone. This silicone mold is used to cast a sacrificial mold using materials such as wax and melted sucrose. The sacrificial mold is destroyed during the final step of fabrication, but the non-transparent silicone mold can be repeatedly used to replicate anatomical models maintaining identical geometry characteristics.

(Figure 1)

\subsection{CAD and channel design}

CAD models of the desired channels were designed using SolidWorks software (SolidWorks, Concord, MA, USA). The models were then converted into stereolithography (STL) format, which is widely used for rapid prototyping and computer-aided design.

Based on the study of T. Chaichana et al. ${ }^{36}$, we used as reference the dimensions of the left coronary artery (LCA). We started with the simple case of a straight channel with two different diameters, $d, 2.5 \mathrm{~mm}$ and $3.5 \mathrm{~mm}$, and evolved to a more complex case of a bifurcation channel, with one inlet (main branch) and two outlets (daughter branches); a $90^{\circ}$ angle was considered between the two daughter branches of the channel. To achieve an approximated geometrical representation of the blood vessels, cross-sections of the daughter branches were made elliptical. These three CAD models are illustrated in Figure 2.

Parallelepiped blocks were introduced in the extremities of the designed CAD models. By adjusting the height of the blocks, the wall thickness of the final model can be controlled. A maximum thickness of $1 \mathrm{~mm}$ was considered in order to get a suitable focal distance to the middle plane of the channels. This technique can also be utile to assure a co-planar system, i.e., a system where the middle planes of all the branches are coincident. In this case, the size of the parallelepiped blocks must compensate the differences in the diameters of the branches.

In addition, we also considered the inclusion of a stenosis, which can be located in different positions in the vessels and have different degrees of occlusion.

(Figure 2)

\subsection{Rapid prototyping}

The CAD models, shown in Figure 2, were used to construct epoxy resin molds by rapid prototyping using solid state stereolithography. The prototyping equipment used was an Accura ${ }^{\circledR} 55$ Plastic from $3 \mathrm{D}$ Systems ${ }^{\circledR}$ with a precision of $0.15 \mathrm{~mm}$. The prototypes, shown in Figures $3 \mathrm{a}$ and $3 \mathrm{~b}$, were used to construct a second mold in non-transparent silicone, Figure $3 c$, at SOLIDtech, Lda.

This second mold can be used to cast a negative sacrificial mold, using materials such as wax and sucrose.

(Figure 3) 


\subsection{Sacrificial mold and PDMS channel}

Two alternative materials were considered for fabricating the sacrificial molds: wax and sucrose.

\subsubsection{Lost-wax casting}

The lost-wax casting method is performed by injecting melted wax ( $353 \mathrm{~K}$ ) into the silicone mold. In the present work, paraffin was used. The wax mold is taken from the nontransparent silicone and placed in a petri dish (Figure 4a). To fabricate the final channels in transparent and elastic material, PDMS is mixed with curing agent in a 5:1 ratio. A Thinky Mixer® ARE-250 was used to prepare the PDMS/curing agent mixture. The mixture is then put into a desiccator connected to a vacuum pump to remove air bubbles. The PDMS is then transferred to the petri dish containing the sacrificial mold and cured at room temperature for 24h (Figure $4 \mathrm{~b}$ ). The wax is removed by melting at $353 \mathrm{~K}$ for $20 \mathrm{~min}$ to obtain the final PDMS channels (Figure 4c).

(Figure 4)

\subsubsection{Lost-sucrose casting}

The fabrication procedure using sucrose is illustrated in Figure 5. The sucrose is caramelized at $433 \mathrm{~K}$ and injected, at this temperature, into the non-transparent silicone mold. Similarly to the lost-wax procedure, the final PDMS mili-scale channels are cured under the same experimental conditions. After the curing step the sucrose is removed by dissolution, in water at room temperature.

(Figure 5)

\subsection{Stenosis fabrication and its implementation in the channels}

For the fabrication of channels with stenosis, a new approach was developed. The stenosis is independently fabricated and inserted into the transparent channel a posteriori. The procedure is illustrated in Figure 6. In Figure 6a four different CAD models of stenoses are represented: with $25 \%, 50 \%$ and $75 \%$ bilateral occlusion and a $50 \%$ unilateral occlusion. Those CAD models (Figure 6a) were used to fabricate the epoxy resin molds by rapid prototyping (Figure $6 \mathrm{~b}$ ) and the non-transparent silicone mold containing different types of stenoses (Figure 6c). The sacrificial mold is, in fact, the negative of the required stenosis, and is inserted into the main 3D PDMS transparent mili-scale channel in the exact position where the stenosis is to be created (Figure 6d). After being positioned in the desired location liquid PDMS is injected using a syringe in the free surrounding space created between the negative mold of the stenosis and the channel walls (Figure 6e). The PDMS is cured at room temperature and the sucrose negative mold is then removed by dissolving it in distilled water (Figure 6f), yielding the final channels with the desired stenosis. In Figure 7 is represented, in detail, the diversity of 3D models, with the sucrose mold inside and after the dissolution process.

(Figure 6)

(Figure 7)

\section{CHARACTERIZATION OF THE IN VITRO MODELS}

The PDMS channels fabricated by lost-wax and lost-sucrose methods were compared and characterized in terms of optical properties and structure. Refractometry and analyses by 
Scanning Electron Microscopy (SEM) and by Energy dispersive X-Ray Spectroscopy (EDS) were performed.

The simplest method to visualize the effect of optical distortion due to the curvature of the solid-liquid interface is to inject a fluid into the channels and placing them over a patterned surface such as the gridlines of a millimeter paper. Three different fluids were used for this purpose: air, water and a water/glycerol mixture. Table 1 shows the relevant properties of the materials used in this study.

The refractive index of the PDMS was measured in an $\mathrm{ABBE}^{\circledR}$ (WYA-1S) digital refractometer, at a controlled temperature of $293 \mathrm{~K}$. A water/glycerol mixture was prepared using $39 \%(\mathrm{w} / \mathrm{w})$ water and $61 \%(\mathrm{w} / \mathrm{w})$ glycerol, in order to match the refractive index of the PDMS ${ }^{37}$ which is essential to eliminate distortion given the curvature of the channel walls. The viscosity of the solution was measured with an Anton Paar ${ }^{\circledR}$ Physica MCR301 Rotational Rheometer.

\section{(Table 1)}

Figure 8 shows the distortion of the gridlines of a sheet of millimeter paper as seen through the 3D mili-scale channels made by lost-wax casting method and by lost-sucrose casting method, when filled with air, water and the water/glycerol mixture.

Channels produced by the lost-sucrose casting method (Figure $8 \mathrm{a} 2$ to $8 \mathrm{c} 2$ ) are clearly better in terms of transparency than those made using wax (Figure $8 \mathrm{a} 1$ to $8 \mathrm{c} 1$ ). Air and water have a much lower refractive index than PDMS and therefore there is no refractive index matching and distortion of the gridlines is clear. On the other hand, the water/glycerol mixture matches the refractive index of the channels material showing no optical distortion. In the channels made by the lost-wax casting method the borders are clearly delimited by wax residues impregnated in the PDMS.

\section{(Figure 8)}

The surface of the mili-scale channels was analyzed using SEM and EDS techniques. The purpose of SEM and EDS analysis is to verify the possible contamination of the model surface by the sacrificial materials (wax and sucrose). SEM allows the detection of small clusters of contaminants over the otherwise smooth surface of PDMS. EDS is used to verify if the chemical composition of the surface differs from that of PDMS.

A SEM image of the surface of the channel obtained using the lost-wax casting method is shown in Figure 9a. The EDS analysis was performed in two different areas: Z1 pointing out a structure that is contaminating the channel and $\mathrm{Z} 2$ representing a clean area. The EDS spectrum shown in Figure $9 \mathrm{~b}$ indicates that the $\mathrm{Z} 2$ area is mostly constituted by silicon, which is one of the constitutive elements of PDMS. The Z1 area has a low silicon count and a high carbon counts indicating the presence of contamination in the PDMS.

(Figure 9)

The mili-scale channels produced using the lost-sucrose casting method exhibit much less contamination, with a smooth surface as shown by SEM image in Figure 10a. The corresponding EDS spectrum confirms silicon (Z3) as the most frequent element (Figure 10b), as expected from the optical distortion analysis.

The results of SEM and EDS have shown that the lost-wax method leaves significant contamination observable by SEM with a change in the chemical composition of the surface detectable by EDS analysis. In the case of lost-sucrose casting method, there are no contaminants observable in the SEM images and the EDS analysis reveals that the surface has, as expected, the composition of PMDS.

(Figure 10)

Figure 11 shows the CAD model of a straight channel and a bifurcated one. The dimensions of the fabricated channels were evaluated by brightfield microscopy (DMI 5000M Leica Microsystems (). Images of the corresponding cross-sections of the channels produced in PDMS are shown in Figure 12. 
(Figure 11)

(Figure 12)

The relevant dimension measured are shown in Table 2, the diameter of the two straight channels, $d$, the inlet diameter of the bifurcated channel, $d_{i}$, and the major and minor diameters of outlet $1\left(d_{o l, M}\right.$ and $\left.d_{o l, m}\right)$ and outlet $2\left(d_{o 2, M}\right.$ and $\left.d_{o 2, m}\right)$.

For the paraffin channels, ten replicates were prepared and their dimensions compared with each other and with the nominal dimensions of the CAD model design. The same procedure was followed for the sucrose channels and fifteen replicates were analyzed. The accuracy of the casting methods was quantified by the mean error defined by:

$$
\epsilon=\frac{\left(\bar{d}-d_{C A D}\right)}{d_{C A D}} \times 100
$$

where $d_{C A D}$ is the reference diameter of the previous geometries designed in CAD software and $\bar{d}$ is the mean diameter of the samples defined by:

$$
\bar{d}=\sum_{k=1}^{n} \frac{d_{k}}{n}
$$

where $d_{k}$ is the diameter of a given sample and $n$ the number of samples.

The reproducibility of the method was assessed through the standard deviation of the samples defined by:

$$
\sigma=\sqrt{\frac{\sum_{k=1}^{n}\left(d_{k}-\bar{d}\right)^{2}}{n-1}}
$$

The geometrical deviation between the channels and the CAD model amounted to a maximum mean error $(\epsilon / \%)$ of $17.7 \%$ (minor axis of outlet 1 ) for paraffin and of $11.3 \%$ (minor axis of outlet 2) for sucrose. It can be concluded, from these values, that the real channels are excellent replicates of the CAD model design, especially if one consider the inherent error associated to the rapid prototyping by solid state stereolithography is approximately $0.15 \mathrm{~mm}$.

The standard deviation varied from 0.07 to $0.31 \mathrm{~mm}$ for the paraffin channels and only 0.02 to $0.16 \mathrm{~mm}$ for the sucrose channels, showing the reproducibility of the channels made by lost-sucrose casting method.

(Table 2)

\section{FLOW CHARACTERIZATION}

The final PDMS channels were used in flow visualization (section 4.1) and $\mu$ PIV (section 4.2) studies to evaluate the ability of the lost-sucrose casting method to fabricate 3D mili-scale channels suitable for optical hemodynamic studies. The fluid used for flow visualization and $\mu$ PIV was the water/glycerol $(39 \% / 61 \% \mathrm{w} / \mathrm{w})$ mixture.

\subsection{Flow visualization}

Flow visualization of the flow patterns were carried out using streak photography with exposure times of the order of $300 \mathrm{~ms}$. For this purpose, the fluid was seeded with $20 \mathrm{ppm}$ $\mathrm{w} / \mathrm{w}$ of $1 \mu \mathrm{m}$ fluorescent polystyrene tracer particles (Nile Red, Molecular Probes, Invitrogen, Ex/Em: 535/575 nm, density: $1.05 \mathrm{~g} / \mathrm{mL}$ ). A syringe pump was used to impose the flow rate in the mili-scale channels. The channels were placed on an inverted epifluorescence microscope (DMI 5000M, Leica Microsystems $\mathrm{GmbH}$ ) and were continuously illuminated by a $100 \mathrm{~W}$ mercury lamp. A filter cube (Leica Microsystems $\mathrm{GmbH}$, excitation filter BP of 530-545 nm, dichroic of $565 \mathrm{~nm}$, and barrier filter of $610-675 \mathrm{~nm}$ ) was used to filter the multichromatic light into the excitation light which reaches the geometries containing the seeded fluid via a microscope objective $(2.5 \mathrm{x}, \mathrm{NA}=0.07)$. Figure 13 illustrates the experimental setup used for flow visualization and $\mu$ PIV analysis. 
The light emitted by the fluorescent tracer particles was imaged through the same objective into the camera DFC350 FX (Leica Microsystems GmbH) in order to capture the particle path-lines, as shown in Figure 14. All images presented here are centered at the midplane of the mili-scale channel. The flow visualization experiments were carried out at a controlled room temperature $(\mathrm{T} \sim 293 \mathrm{~K})$ for a wide range of flow rates $(100 \leq \mathrm{Q} \leq 1000 \mathrm{ml} \mathrm{h}$ $\left.{ }^{1}\right)$.

\section{(Figure 14)}

Using an index-matched fluid eliminates most of the optical distortion caused by the channel curvature and reliable visualizations of the flow patterns can be performed. This visualization was a preliminary study to carry out a reliable $\mu$ PIV analysis.

\section{2 $\mu$ PIV analysis}

Measurements of the velocity field were carried out using $\mu$ PIV. For that purpose, the fluid was seeded with the same fluorescent microparticles used in the flow visualization. The generic imaging setup used is similar to that described in the previous section except for the light source. The light source used for $\mu$ PIV is a doubled pulsed Nd:YAG (yttrium aluminum garnet) laser (Dual Power 65-15, Dantec Dynamics) with a wavelength of $532 \mathrm{~nm}$. The time interval between pulses was adjusted in the range of $100 \leq \Delta \mathrm{t}[\mu \mathrm{s}] \leq 1000$, such that particle displacement was about $25 \%$ the size of the interrogation area, which was selected according to the velocity of the flow and the objective used. For each flow rate, 100 pairs of images were acquired using a digital camera (Flow Sense 4M, Dantec Dynamics) with a resolution of 2048 x 2048 pixels and running in double frame mode. The images were post-processed to obtain the velocity vector map using DYNAMIC STUDIO V2.3 software (Dantec Dynamics) based on ensemble averaging the 100 pairs of images. All images were acquired at the midplane of the mili-scale channel.

Two different geometries were chosen for $\mu \mathrm{PIV}$ data analysis, a bifurcated channel with a stenosis in one of the daughter branches ( $25 \%$ occlusion), Figures 15, 16 and 17, and a straight channel containing a stenosis (50\% occlusion), Figures 18 and 19.

(Figure 15)

Figure 16 shows the velocity field normalized by the mean velocity in the inlet channel. Note that the normalized velocity is lower in the daughter branch that contains the stenosis due to the higher pressure drop along that branch.

(Figure 16)

Figure 17 represents the velocity field normalized by the mean velocity in the stenotic branch of the bifurcated channel. As described above the red arrows show the flow direction.

(Figure 17)

Figure 18 shows a straight channel containing the detail of a stenosis with a $50 \%$ occlusion. The rectangular regions represented, I and II, are those where the $\mu$ PIV experiments were carried out.

\section{(Figure 18)}

In Figure 19, the corresponding velocity fields are illustrated: I) region of the velocity field upstream of the stenosis (Figure 19a) and II) in the stenosis region (Figure 19b). For better understanding the velocity field was overlapped on a brightfield image at the same site where the $\mu$ PIV was carried out. 
The mean velocity is higher in the stenosis due to the smaller diameter, as expected. The velocity profiles along the straight channel are shown in Figure 20. This representation shows that the flow, in the largest diameter area, is developed upstream the stenosis and starts accelerating as it approach the stenosis region. In the stenosis region the velocity profiles are more elongated and the peak velocity is higher as expected.

(Figure 20)

\section{CONCLUSION}

The objective of this study was to find a good fabrication method of 3D mili-scale channels for hemodynamic studies. The most common methods for fabrication of in vitro models of mili-scale arteries rely on the use of sacrificial template techniques. However, the removal process of these materials is usually done by melting. This heat removal process is known to leave residues in the walls of the final model which compromise the optical experiments and jeopardize the credibility of the final results. One of the most employed methods in the literature, the lost-wax casting method, was tested and compared to a novel process created to surpass the known limitations of the usual fabrication methods. The developed process relies on the use of a material that could be removed without the need of high temperatures, but could be injected in any format silicon mold. The material used was sucrose, and the method was denominated the "lost-sucrose" casting method. It was shown that, for fabricating 3D PDMS mili-scale channels, the developed method not only fulfilled all the requirements for this type of process but also was better than the common methods found in literature. This method enables the fabrication of mili-scale channels with excellent optical properties, in terms of transparency and very low level of optical distortion.

These findings are explained by the much less level of contamination of the channels during the fabrication process with sucrose. The low level of contamination is explained by the removal process used. In the lost-sucrose casting method, the removal is by dissolution in water at room temperature while in the lost-wax casting method, as in other methods, typically removal is done by melting at high temperatures which leaves residues in the walls of the channels.

In terms of $\mu \mathrm{PIV}$ it was possible to study the velocity field and extract the velocity profiles along the $3 \mathrm{D}$ mili-scale channels. The $\mu \mathrm{PIV}$ technique is often used in micro and macro-channels and this study proves that it is possible to apply with accuracy this technique to the 3D mili-scale channels fabricated with the sucrose method.

This work will allow a more realistic approach for future hemodynamic studies that focus on atherosclerosis in mili-scale arteries.

\section{ACKNOWLEDGMENTS}

The authors gratefully acknowledge the funding by Fundação para a Ciência e Tecnologia, FCT (Portugal), and FEDER under projects PTDC/EME-MFE/102974/2008 and PTDC/EME-MFE/099109/2008. SEM and EDS were performed at CEMUP facilities (funded by FCT projects REEQ/1062/CTM/2005 and REDE/1512/RME/2005).

\section{REFERENCES}

1. Lozano R, Naghavi M, Foreman K, Lim S, et al., Global and regional mortality from 235 causes of death for 20 age groups in 1990 and 2010: a systematic analysis for the Global Burden of Disease Study 2010, The Lancet 380:2095-2128, 2012.

2. Chatzizisis YS, Coskun AU, Jonas M, Edelman ER, et al., Role of Endothelial Shear Stress in the Natural History of Coronary Atherosclerosis and Vascular Remodeling: Molecular, Cellular, and Vascular Behavior, Journal of the American College of Cardiology 49:23792393, 2007. 
3. Bale-Glickman J, Selby K, Saloner D, Savas O, Experimental flow studies in exact-replica phantoms of atherosclerotic carotid bifurcations under steady input conditions, Journal of biomechanical engineering 125:38-48, 2003.

4. Shaaban AM, Duerinckx AJ, Wall shear stress and early atherosclerosis: a review, AJR. American journal of roentgenology 174:1657-1665, 2000.

5. Zurauskas E, Usinskiene J, Gaigalaite V, Blanc R, et al., [Models of intracranial aneurysms for angiographic imaging modalities. A technical note], Medicina 43:562-567, 2007.

6. Baek H, Jayaraman MV, Richardson PD, Karniadakis GE, Flow instability and wall shear stress variation in intracranial aneurysms, Journal of the Royal Society, Interface / the Royal Society 7:967-988, 2010.

7. Valen-Sendstad K, Mardal KA, Mortensen M, Reif BA, et al., Direct numerical simulation of transitional flow in a patient-specific intracranial aneurysm, J Biomech 44:2826-2832, 2011.

8. Ford MD, Piomelli U, Exploring high frequency temporal fluctuations in the terminal aneurysm of the basilar bifurcation, Journal of biomechanical engineering 134:091003, 2012.

9. Brunette J, Mongrain R, Laurier J, Galaz R, et al., 3D flow study in a mildly stenotic coronary artery phantom using a whole volume PIV method, Medical engineering \& physics 30:1193-1200, 2008.

10. Lorthois S, Stroud-Rossman J, Berger S, Jou LD, et al., Numerical simulation of magnetic resonance angiographies of an anatomically realistic stenotic carotid bifurcation, Ann Biomed Eng 33:270-283, 2005.

11. Shalman E, Rosenfeld M, Dgany E, Einav S, Numerical modeling of the flow in stenosed coronary artery. The relationship between main hemodynamic parameters, Computers in Biology and Medicine 32:329-344, 2002.

12. Bertolotti C, Deplano V, Fuseri J, Dupouy P, Numerical and experimental models of postoperative realistic flows in stenosed coronary bypasses, J Biomech 34:1049-1064, 2001.

13. Ku JP, Elkins CJ, Taylor CA, Comparison of CFD and MRI flow and velocities in an in vitro large artery bypass graft model, Ann Biomed Eng 33:257-269, 2005.

14. Kabinejadian F, Cui F, Zhang Z, Ho P, et al., A Novel Carotid Covered Stent Design: In Vitro Evaluation of Performance and Influence on the Blood Flow Regime at the Carotid Artery Bifurcation, Annals of Biomedical Engineering 41:1990-2002, 2013.

15. Shahcheraghi N, Dwyer HA, Cheer AY, Barakat AI, et al., Unsteady and threedimensional simulation of blood flow in the human aortic arch, Journal of biomechanical engineering 124:378-387, 2002.

16. Kobayashi MH, Pereira JC, A computational method for solving arbitrary twodimensional physiological flows, Journal of biomechanical engineering 116:315-317, 1994.

17. Barakat AI, Cheng ET, Numerical simulation of fluid mechanical disturbance induced intravascular stents, in Proceedings of ICMMB-11, CMMB-11: International Conference on Mechanics in Medicine and Biology, Maui, Hawaii,2000.

18. Braunmuller S, Schmid L, Franke T, Dynamics of red blood cells and vesicles in microchannels of oscillating width, Journal of physics. Condensed matter : an Institute of Physics journal 23:184116, 2011.

19. Einav S, Berman HJ, Fuhro RL, DiGiovanni PR, et al., Measurement of velocity profiles of red blood cells in the microcirculation by laser doppler anemometry (LDA), Biorheology 12:207-210, 1975.

20. Ravnic DJ, Zhang Y-Z, Tsuda A, Pratt JP, et al., Multi-image particle tracking velocimetry of the microcirculation using fluorescent nanoparticles, Microvascular Research 72:27-33, 2006.

21. Sugii Y, Okuda R, Okamoto K, Madarame H, Velocity measurement of both red blood cells and plasma of in vitro blood flow using high-speed micro PIV technique, Meas. Sci. Technol. 16:1126-1130, 2005.

22. Santiago JG, Wereley ST, Meinhart CD, Beebe DJ, et al., A particle image velocimetry system for microfluidics, Experiments in Fluids 25:316-319, 1998.

23. Lima R, Wada S, Tsubota K, Yamaguchi T, Confocal micro-PIV measurements of threedimensional profiles of cell suspension flow in a square microchannel, Meas. Sci. Technol. 17:797-808, 2006.

24. Park JS, Choi CK, Kihm KD, Optically sliced micro-PIV using confocal laser scanning microscopy (CLSM), Experiments in Fluids 37:105-119, 2004. 
25. Burgmann S, Grosse S, Schroder W, Roggenkamp J, et al., A refractive index-matched facility for fluid-structure interaction studies of pulsatile and oscillating flow in elastic vessels of adjustable compliance, Experiments in Fluids 47:865-881, 2009.

26. de Zelicourt D, Pekkan K, Kitajima H, Frakes D, et al., Single-step stereolithography of complex anatomical models for optical flow measurements, Journal of biomechanical engineering 127:204-207, 2005.

27. de Zelicourt DA, Pekkan K, Wills L, Kanter K, et al., In vitro flow analysis of a patientspecific intraatrial total cavopulmonary connection, The Annals of thoracic surgery 79:20942102, 2005.

28. Gailloud P, Muster M, Piotin M, Mottu F, et al., In vitro models of intracranial arteriovenous fistulas for the evaluation of new endovascular treatment materials, AJNR. American journal of neuroradiology 20:291-295, 1999.

29. Wetzel SG, Ohta M, Handa A, Auer JM, et al., From patient to model: stereolithographic modeling of the cerebral vasculature based on rotational angiography, AJNR. American journal of neuroradiology 26:1425-1427, 2005.

30. Knox K, Kerber CW, Singel SA, Bailey MJ, et al., Rapid prototyping to create vascular replicas from CT scan data: making tools to teach, rehearse, and choose treatment strategies, Catheterization and cardiovascular interventions : official journal of the Society for Cardiac Angiography \& Interventions 65:47-53, 2005.

31. Allard L, Soulez G, Chayer B, Treyve F, et al., Multimodality vascular imaging phantoms: a new material for the fabrication of realistic 3D vessel geometries, Med Phys 36:3758-3763, 2009.

32. Smith RF, Rutt BK, Holdsworth DW, Anthropomorphic carotid bifurcation phantom for MRI applications, Journal of magnetic resonance imaging : JMRI 10:533-544, 1999.

33. Cloutier G, Soulez G, Qanadli SD, Teppaz P, et al., A multimodality vascular imaging phantom with fiducial markers visible in DSA, CTA, MRA, and ultrasound, Med Phys 31:1424-1433, 2004.

34. Geoghegan PH, Buchmann NA, Spence CJT, Moore S, et al., Fabrication of rigid and flexible refractive-index-matched flow phantoms for flow visualisation and optical flow measurements, Experiments in Fluids 52:1331-1347, 2012.

35. Lee J, Paek J, Kim J, Sucrose-based fabrication of 3D-networked, cylindrical microfluidic channels for rapid prototyping of lab-on-a-chip and vaso-mimetic devices, Lab on a Chip 12:2638-2642, 2012.

36. Chaichana T, Sun Z, Jewkes J, Computation of hemodynamics in the left coronary artery with variable angulations, J Biomech 44:1869-1878, 2011.

37. Buchmann NA, Atkinson C, Jeremy MC, Soria J, Tomographic particle image velocimetry investigation of the flow in a modeled human carotid artery bifurcation, Experiments in Fluids 50:1131-1151, 2011. 


\section{Figure Captions}

Fig.1 Schematic diagram of the fabrication process.

Fig.2 CAD models used in the rapid prototyping by stereolithography: a) straight channel with $d=2.5 \mathrm{~mm}$, b) straight channel with $d=3.5 \mathrm{~mm}$ and c) bifurcated channel with a main branch diameter of $3.5 \mathrm{~mm}$.

Fig.3 Epoxy resin mold made by stereolithography of: a) two straight channels, b) bifurcated channel and c) nontransparent silicone mold containing the various geometries.

Fig.4 Fabrication technique of the final PDMS channels using the lost-wax casting method: a) negative mold made of paraffin, b) PDMS channel after the curing stage and before the wax removal step by melting and c) final 3D PDMS channel.

Fig.5 Fabrication technique of the final PDMS channels using the lost-sucrose casting method: a) negative mold made of sucrose, b) PDMS channel after the curing stage before the sucrose removal step by dissolution and c) final 3D PDMS channel.

Fig.6 Stenoses fabrication and its implementation in the channels, using the lost-sucrose casting method: a) CAD models, b) epoxy resin molds, c) non-transparent silicone mold containing the different stenoses geometries, d) sacrificial sucrose mold in the desired position, e) injection of the PDMS and f) final 3D PDMS channel with the desired stenosis. The larger diameter of the stenoses models is the same as the diameter of the channels, $2.5 \mathrm{~mm} \leq D \leq$ $3.5 \mathrm{~mm}$. The length of the stenoses models varies between $5 \mathrm{~mm}$ and $15 \mathrm{~mm}$.

Fig.7 3D PDMS mili-scale channels fabricated by the lost-sucrose casting method: a1 to d1) before the sucrose dissolution and a2 to d2) after the sucrose dissolution.

Fig.8 Distortion of gridlines seen through the 3D mili-scale PDMS channel, fabricated by lost-wax casting method (top) and by lost-sucrose casting method (bottom), when the channel is filled with: a) air, b) water and c) water/glycerol.

Fig.9 Surface analysis of a mili-channel fabricated by the lost-wax casting method: a) SEM image showing the location of the surfaces analysed and b) EDS Spectrum showing the different element counts in the Z1 and Z2 areas.

Fig.10 Surface analysis of a mili-channel fabricated by the lost-sucrose casting method: a) SEM image showing the location of the surface analysed and b) EDS Spectrum showing the different element counts in the Z3 area.

Fig.11 CAD models: a) straight channel with $d=2.50 \mathrm{~mm}$; b) straight channel with $d=3.50 \mathrm{~mm}$; c) bifurcated channel, with one inlet, $\mathrm{d}_{\mathrm{i}}=3.50 \mathrm{~mm}$, and two outlets (daughter branches) with ellipse shaped cross section (with major diameters $d_{o l, M}=d_{o 2, M}=3.50 \mathrm{~mm}$ and minor diameters $d_{o l, m}=d_{o 2, m}=2.78 \mathrm{~mm}$ ).

Fig.12 Brightfield visualization of the mili-scale channels cross-sections with corresponding diameters: a) inlet, b) outlet 1, c) outlet 2, d) and e) of two different straight channels.

Fig.13 Setup for $\mu$ PIV analysis.

Fig.14 Flow visualization, using water/glycerol, in channels fabricated by the lost-sucrose casting method: a) $Q=100$ $\mathrm{mL} \cdot \mathrm{h}^{-1}$ (50\% unilateral occlusion), b) $Q=500 \mathrm{~mL} \cdot \mathrm{h}^{-1}$ (75\% occlusion), c) $Q=200 \mathrm{~mL} \cdot \mathrm{h}^{-1}$ (bifurcated channel) and d) $Q=1000 \mathrm{~mL} \cdot \mathrm{h}^{-1}$ (straight channel without stenosis). 
Fig.15 Bifurcated channel with a stenosis (25\% occlusion) for $\mu$ PIV analysis. Details: I) the bifurcated area an II) the stenosis area.

Fig.16 Normalized velocity field in the bifurcated 3D mili-scale channel at: a) $Q=600 \mathrm{~mL} \cdot \mathrm{h}^{-1}$, b) $Q=1200 \mathrm{~mL} \cdot \mathrm{h}^{-1}$ and c) $Q=5000 \mathrm{~mL} \cdot \mathrm{h}^{-1}$. The red arrows show the flow direction.

Fig.17 Normalized velocity field in the stenosis region (25\% occlusion) at: a) $Q=600 \mathrm{~mL} \cdot \mathrm{h}^{-1}$, b) $Q=1200 \mathrm{~mL} \cdot \mathrm{h}^{-1}$ and c) $Q=5000 \mathrm{~mL} \cdot \mathrm{h}^{-1}$.

Fig.18 CAD representation of a straight channel containing a stenosis, highlighting the detail of stenosis with $50 \%$ occlusion and two regions where the $\mu$ PIV analysis was performed: I) upstream the stenosis and II) in the stenosis.

Fig.19 Velocity fields overlapped on brightfield images representing two different regions of the straight channel for $Q=1000 \mathrm{~mL} \cdot \mathrm{h}^{-1}:$ a) upstream of the stenosis and b) in the stenosis (50\% occlusion). The red arrows represent the flow direction.

Fig.20 Experimental and theoretical $\left(Q=1047 \mathrm{~mL} \cdot \mathrm{h}^{-1}\right)$ velocity profiles measured along the midplane of the straight channel highlighting the stenosis region (50\% occlusion).

\section{Table Captions}

Table 1. Relevant properties of the materials used in this study at a controlled temperature of $293 \mathrm{~K}$.

Table 2. Evaluation of cross-sections dimensions of the final 3D PDMS mili-scale channels. 
3D print (epoxy mold)


3D PDMS Channel

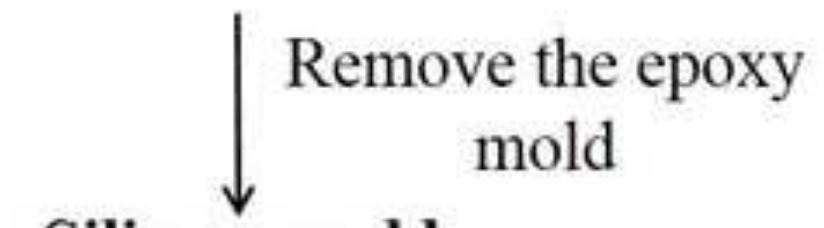

Silicone mold

(non-transparent)

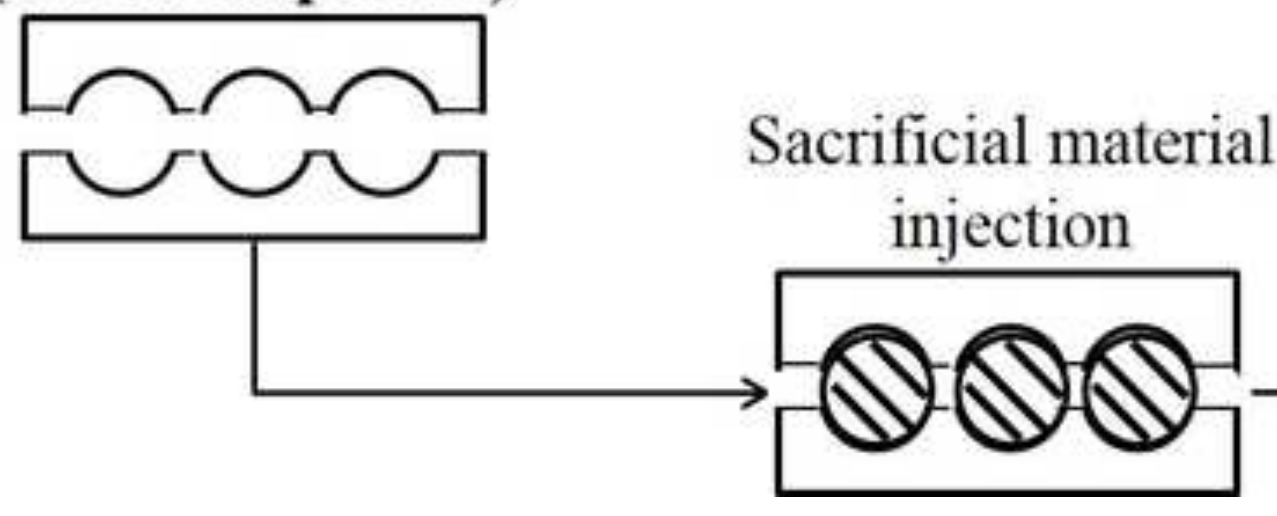



Sacrificial Mold 


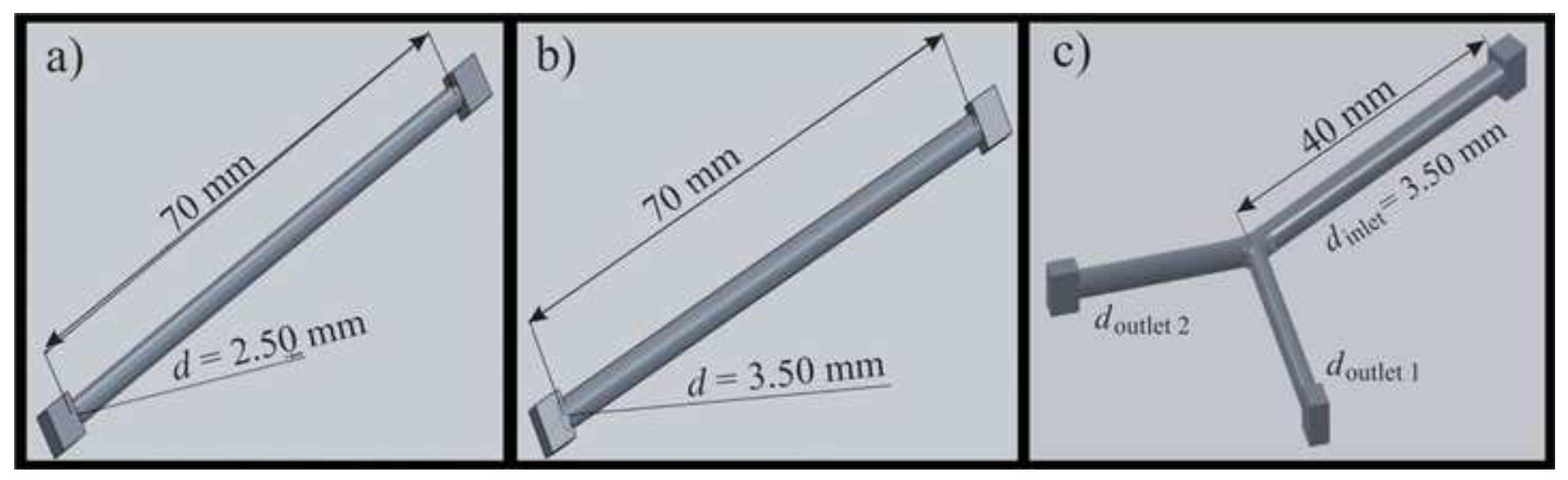



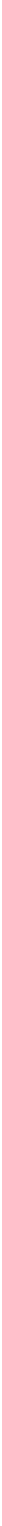

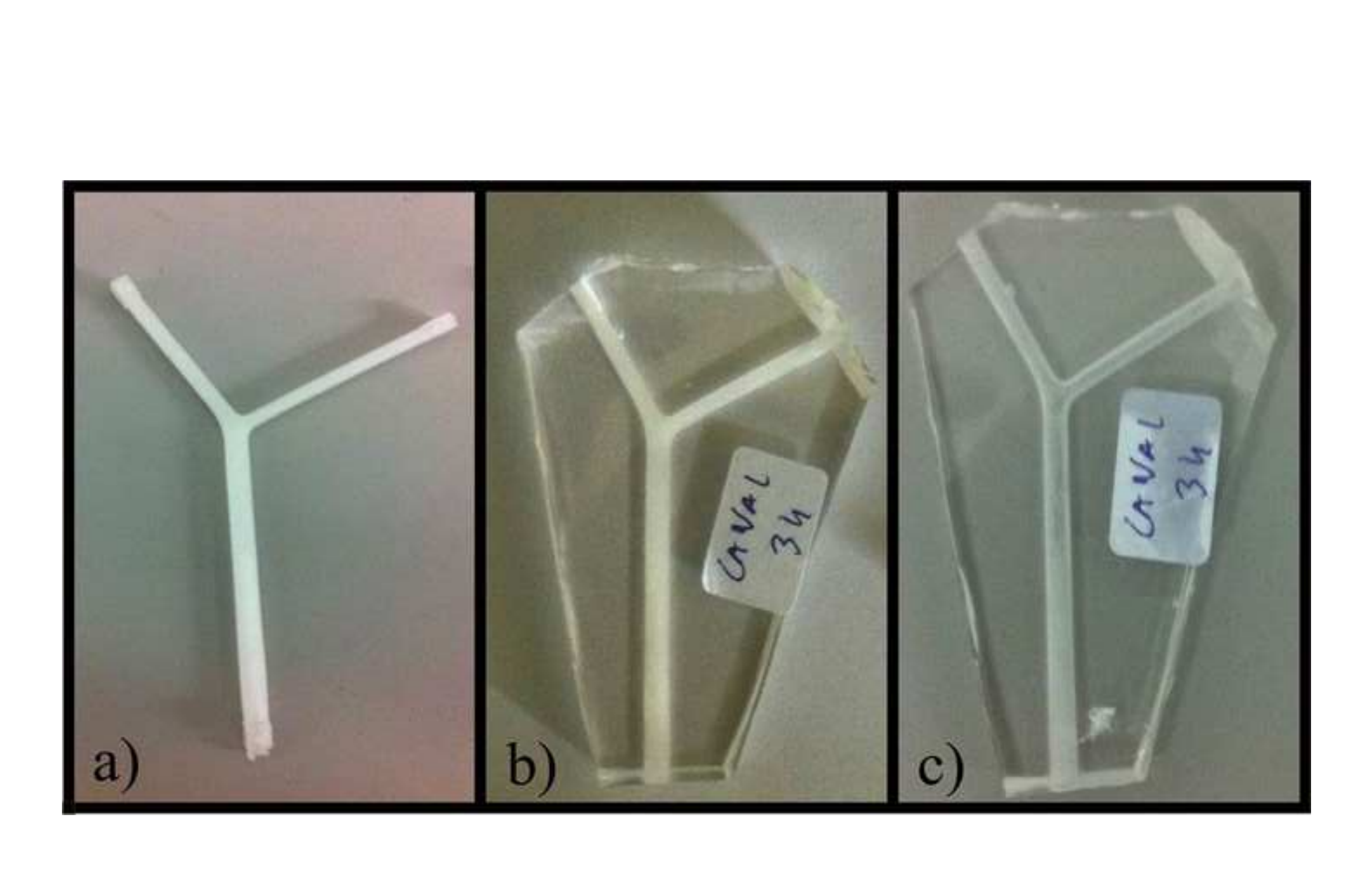

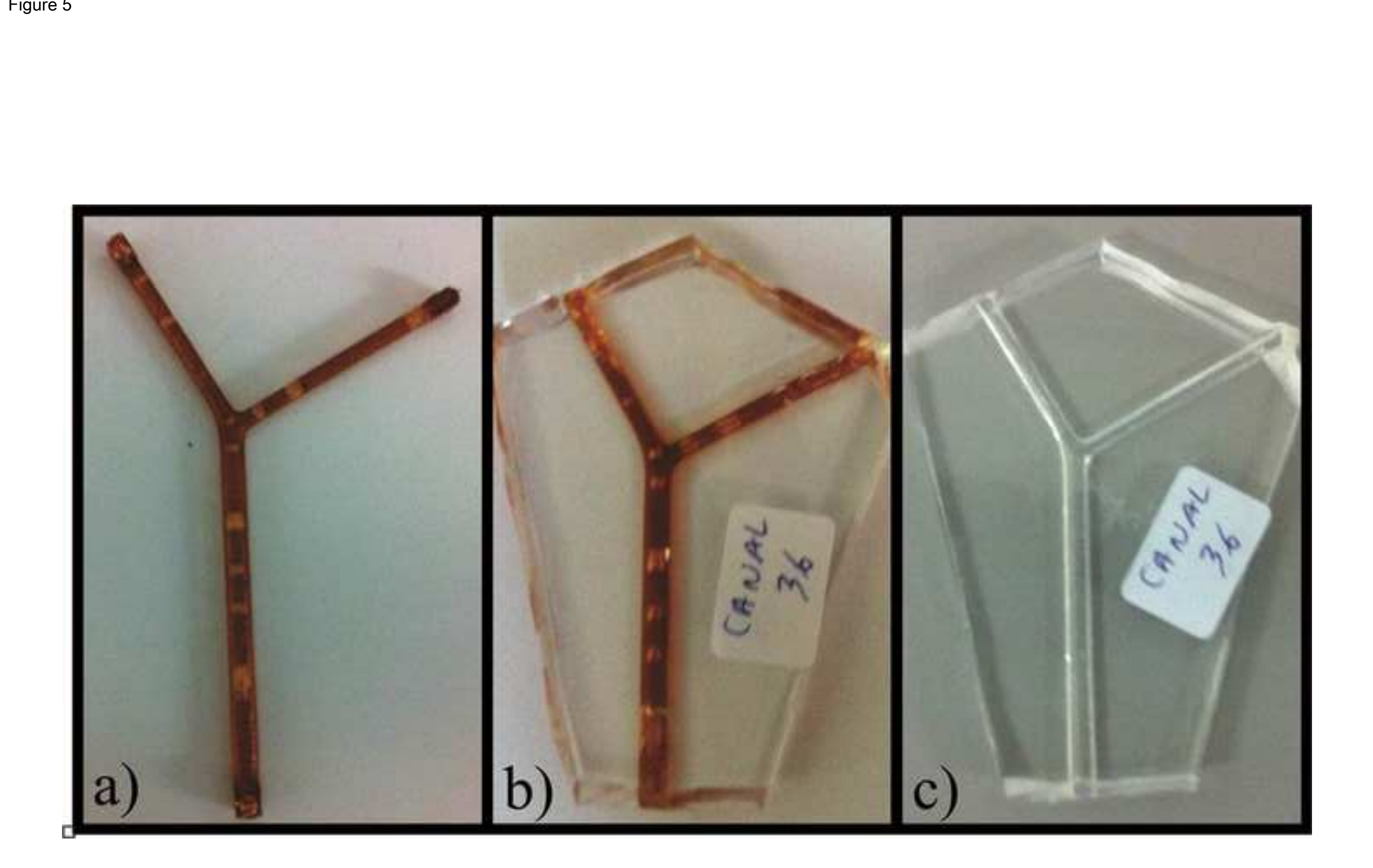


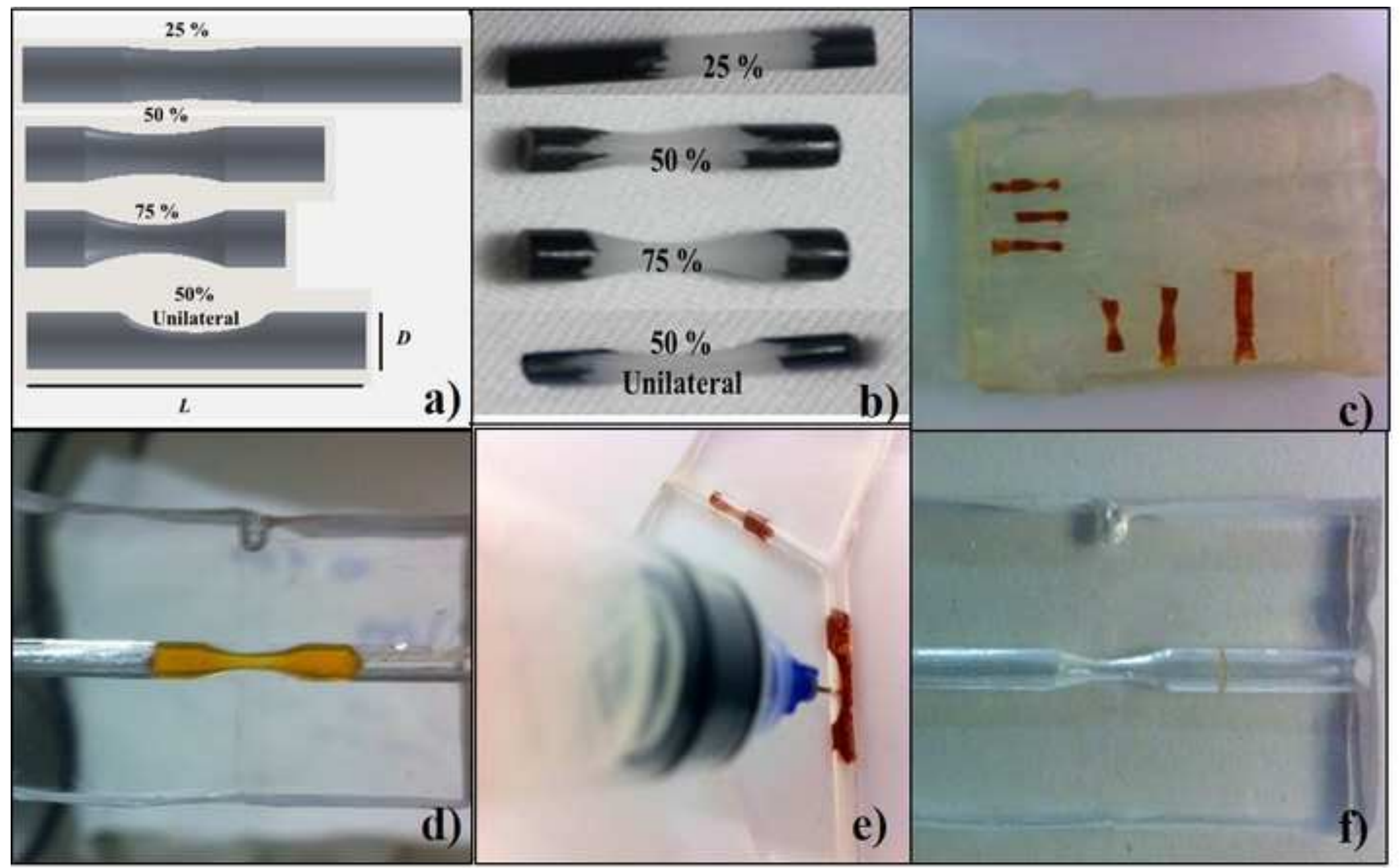




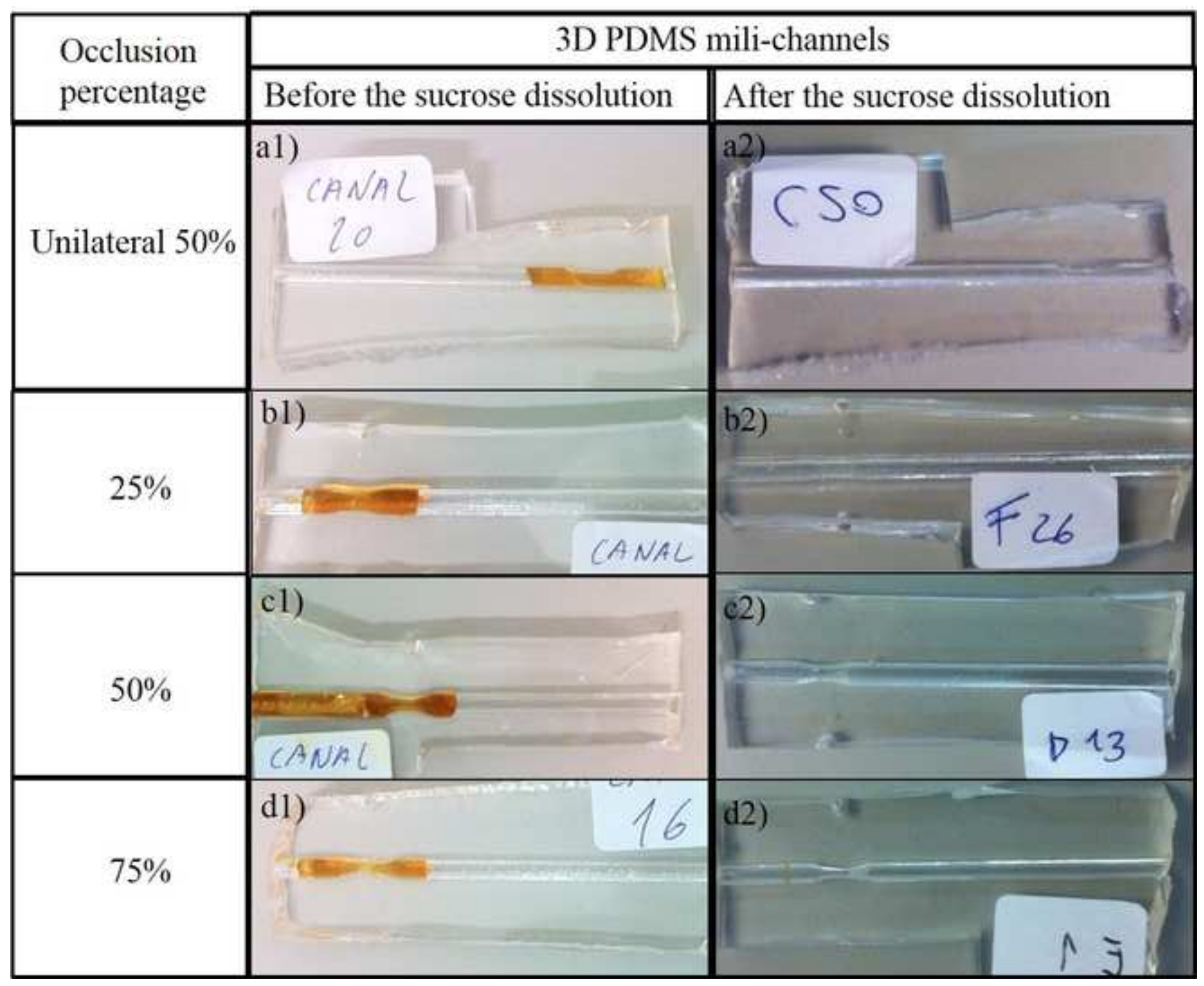




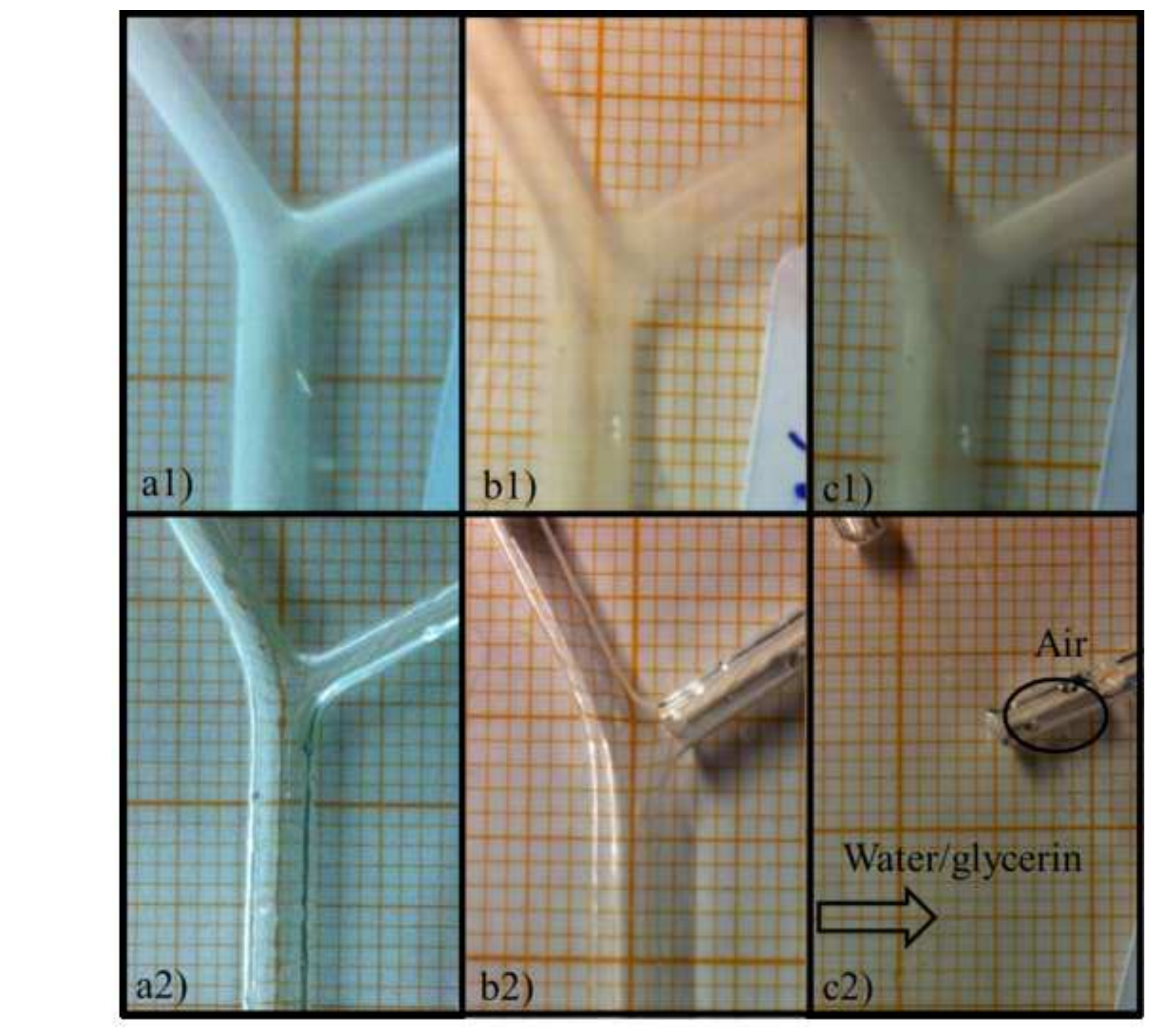




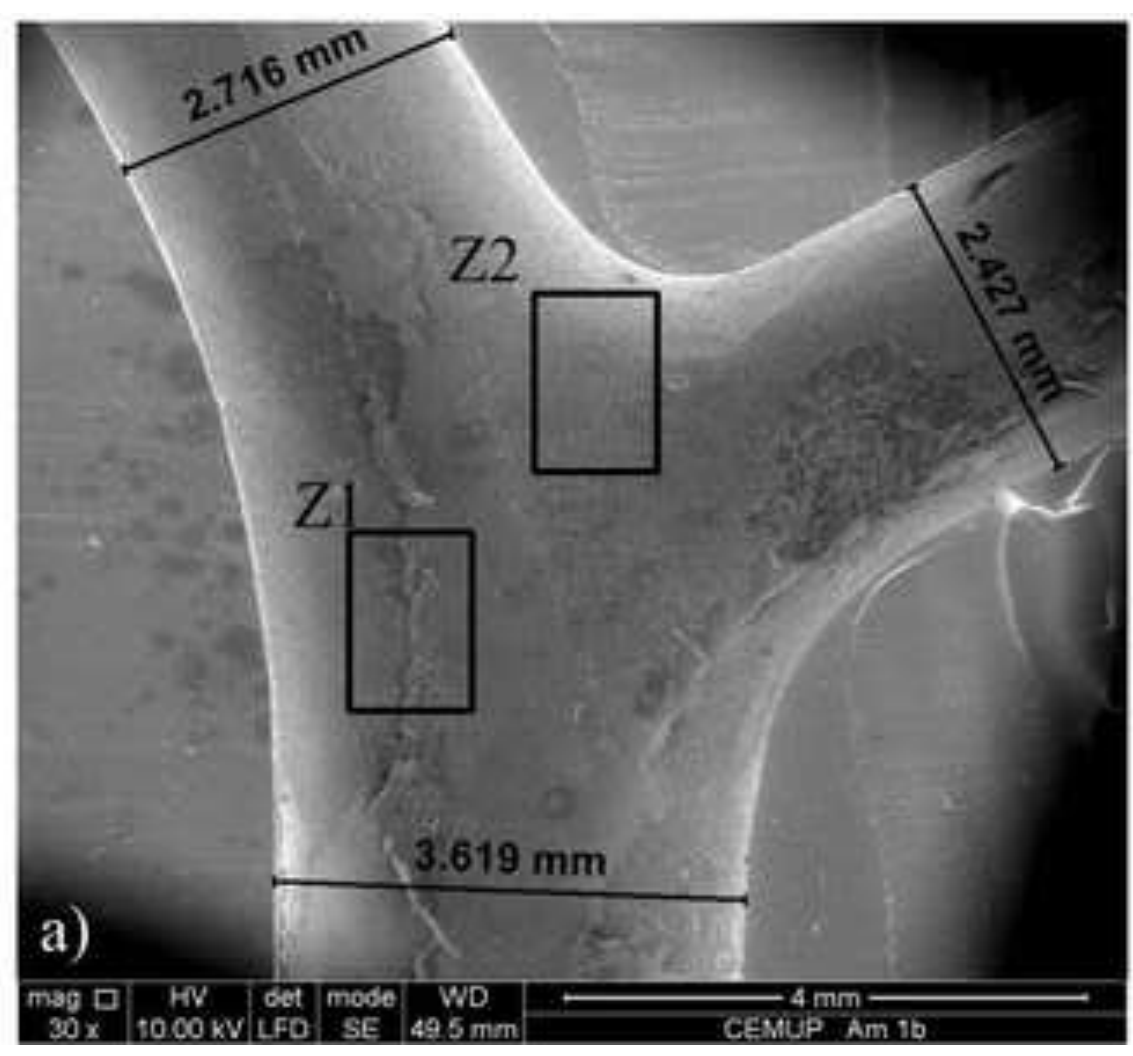

b)

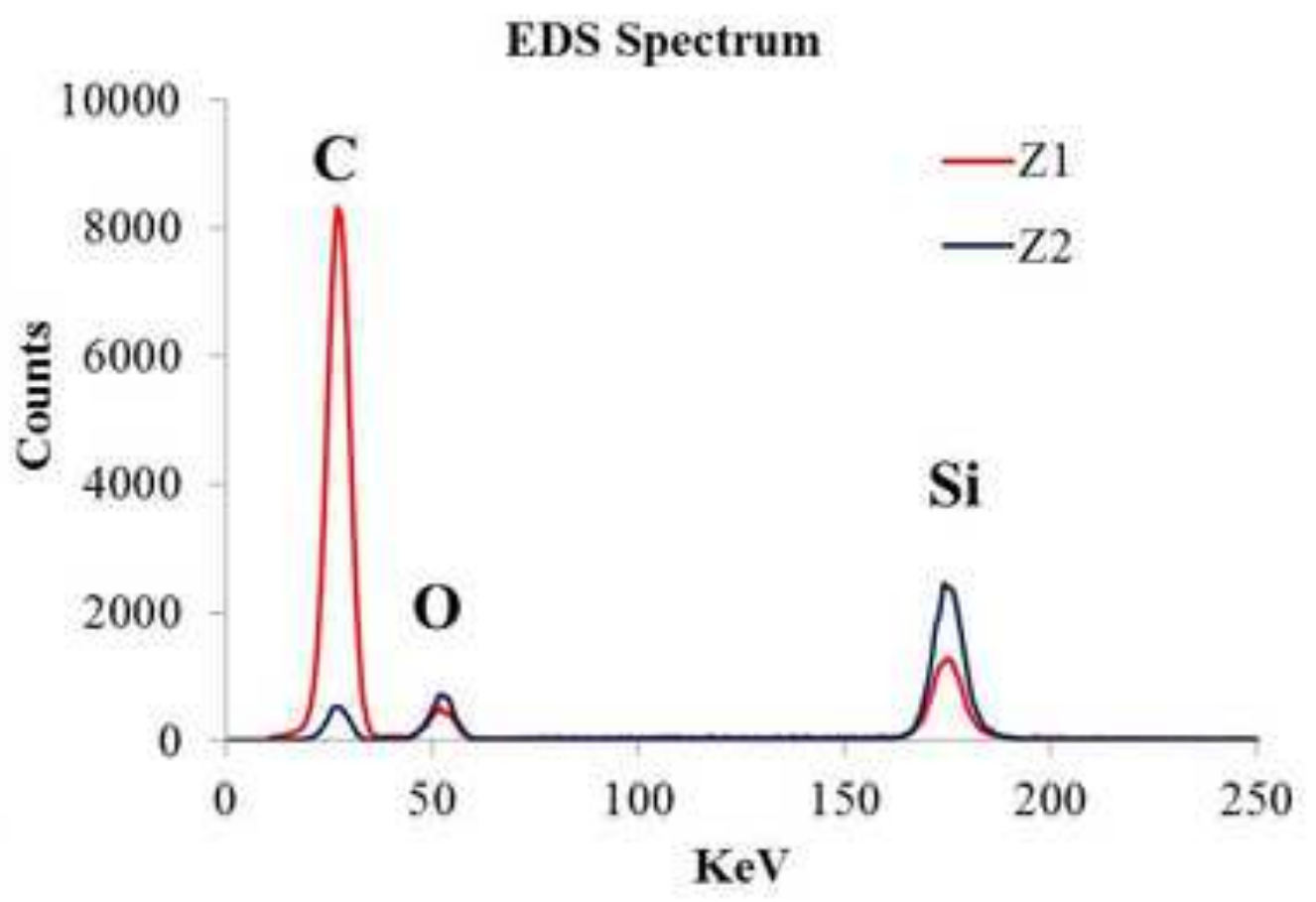




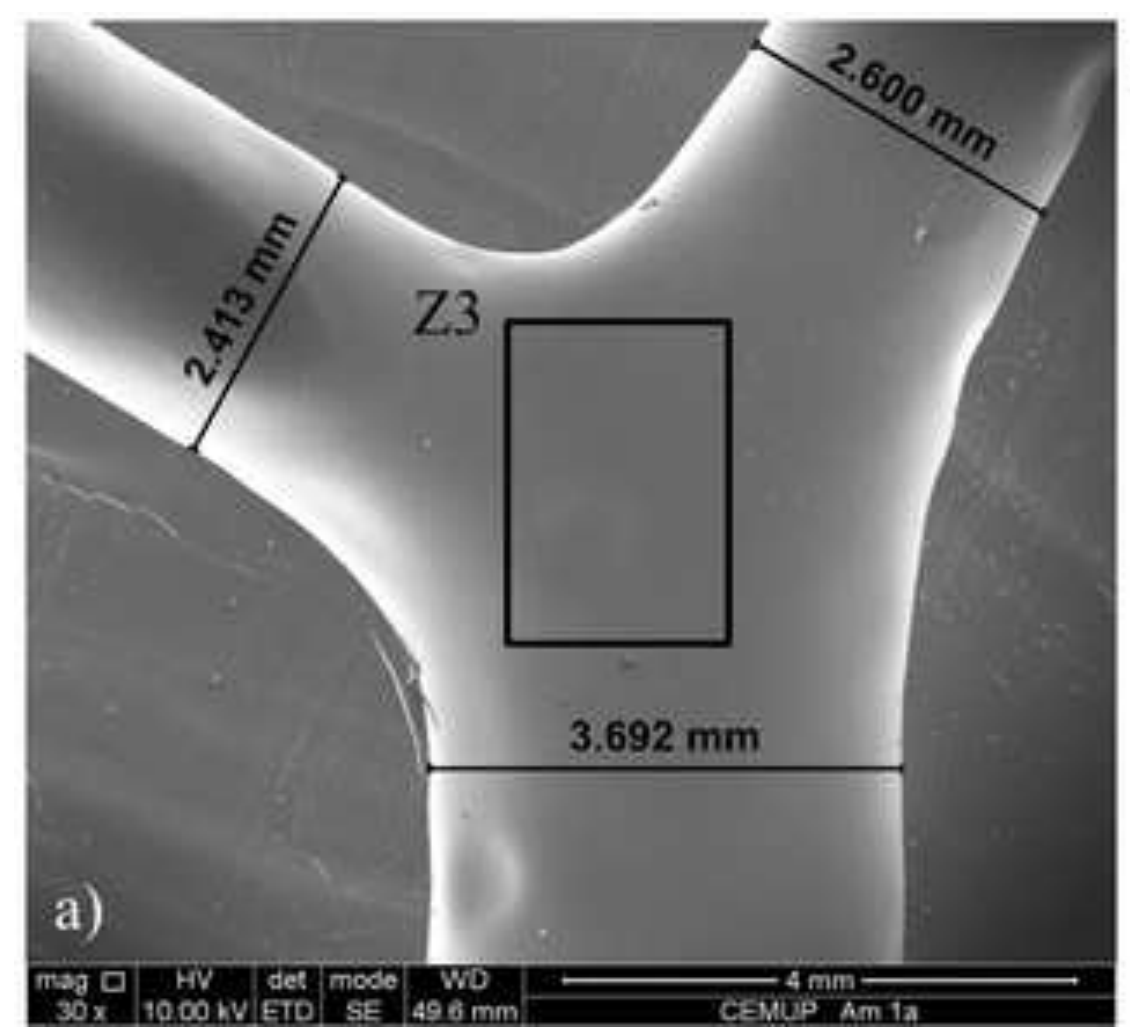

b)

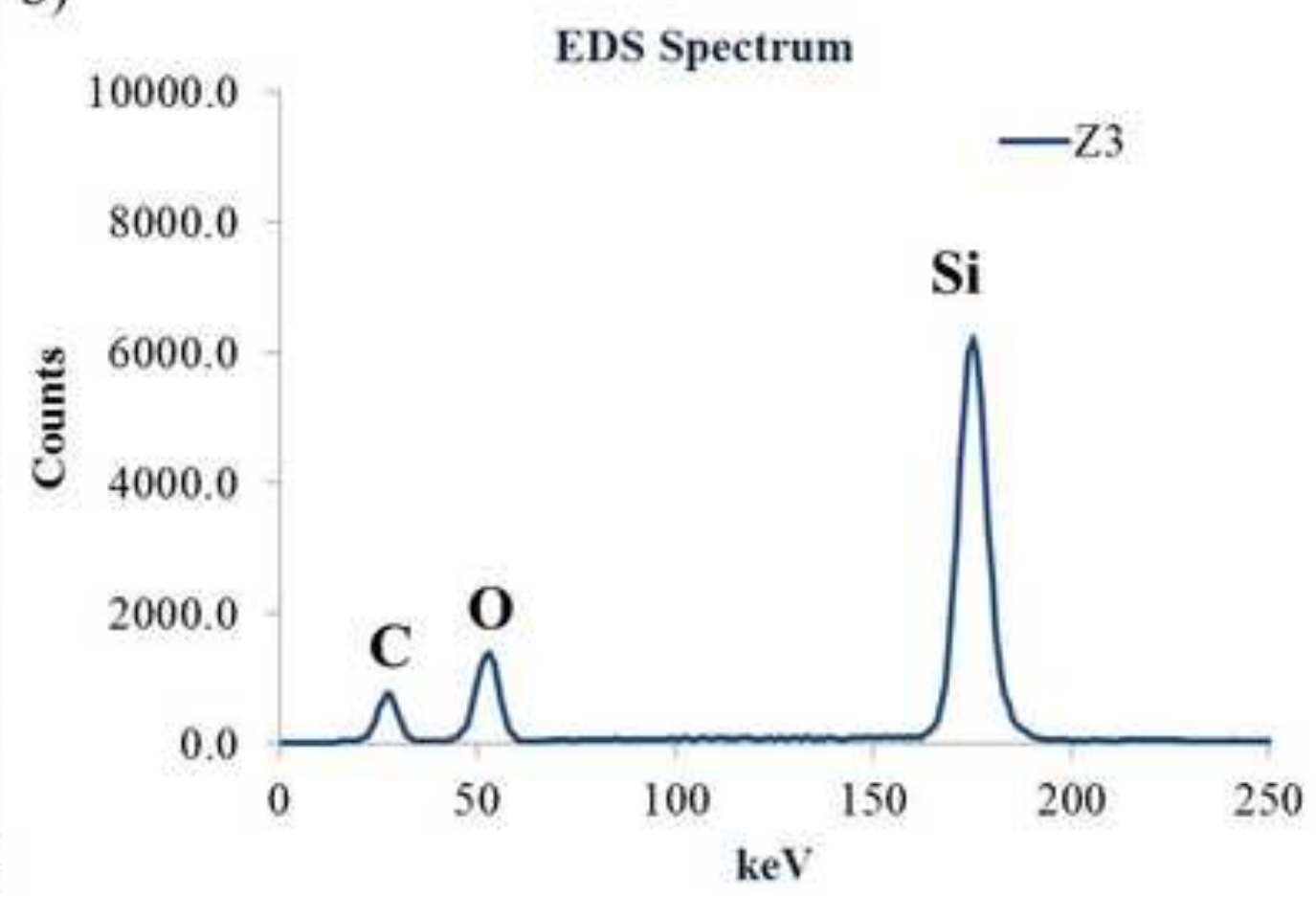




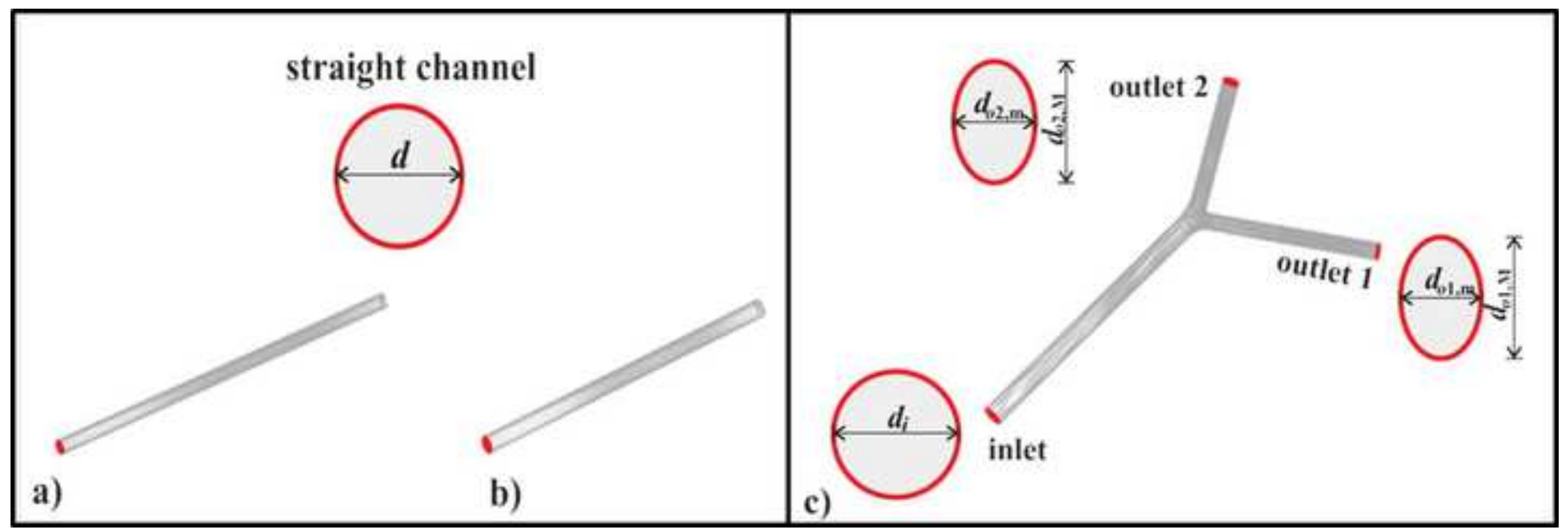



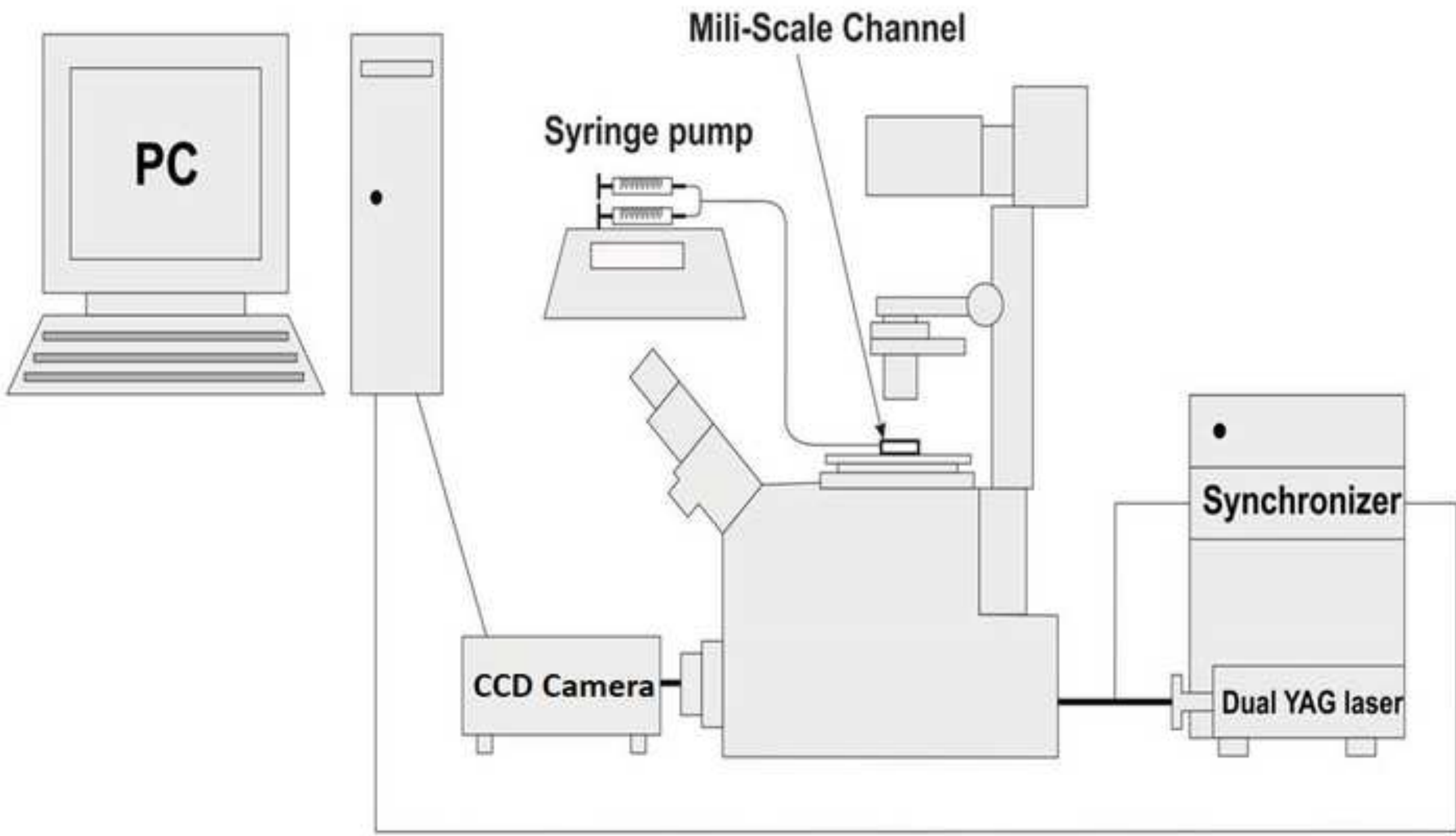
a)

$-$

y

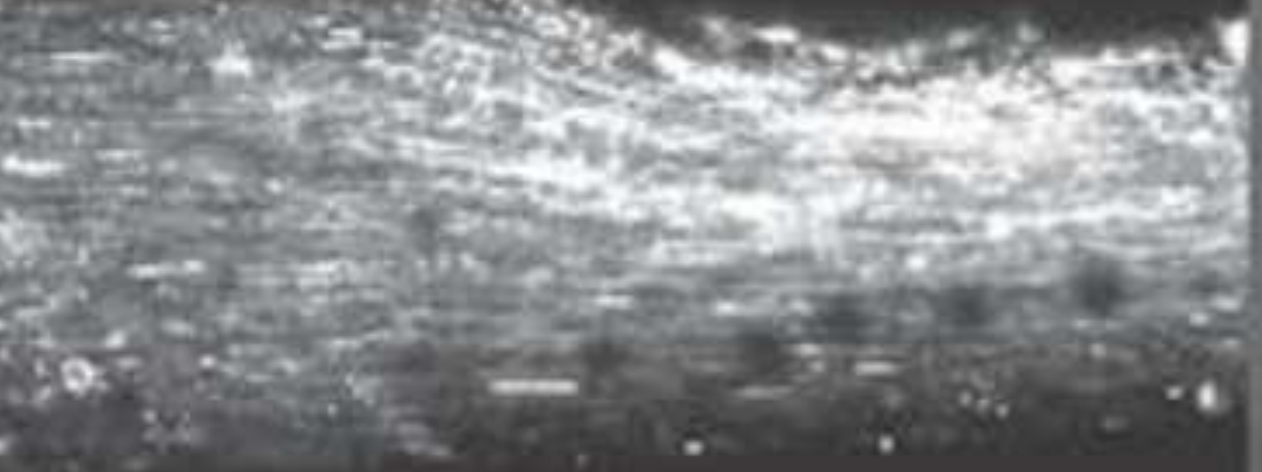

c)

.

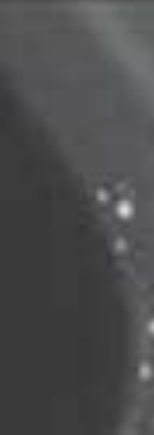

b)

d)
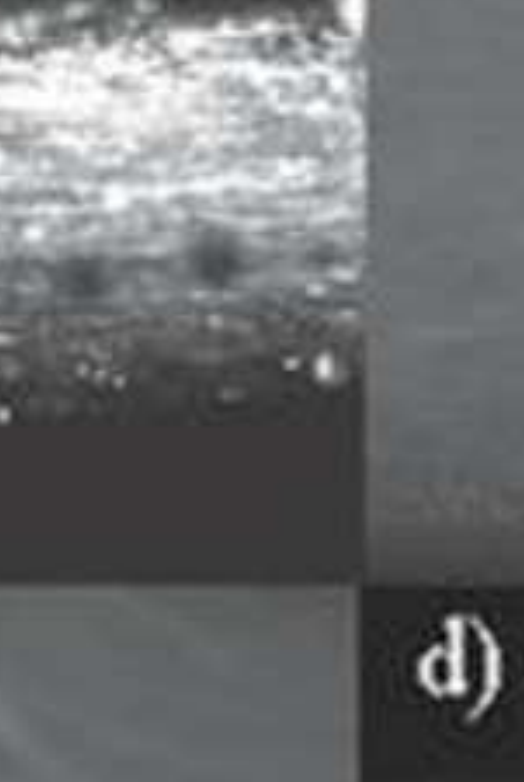

$*$

.

$\sqrt{20}$

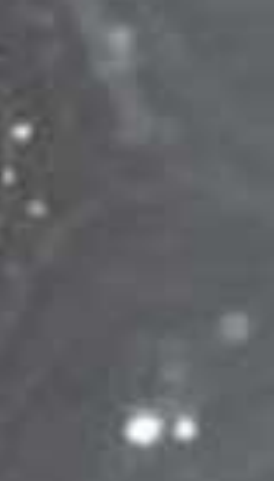




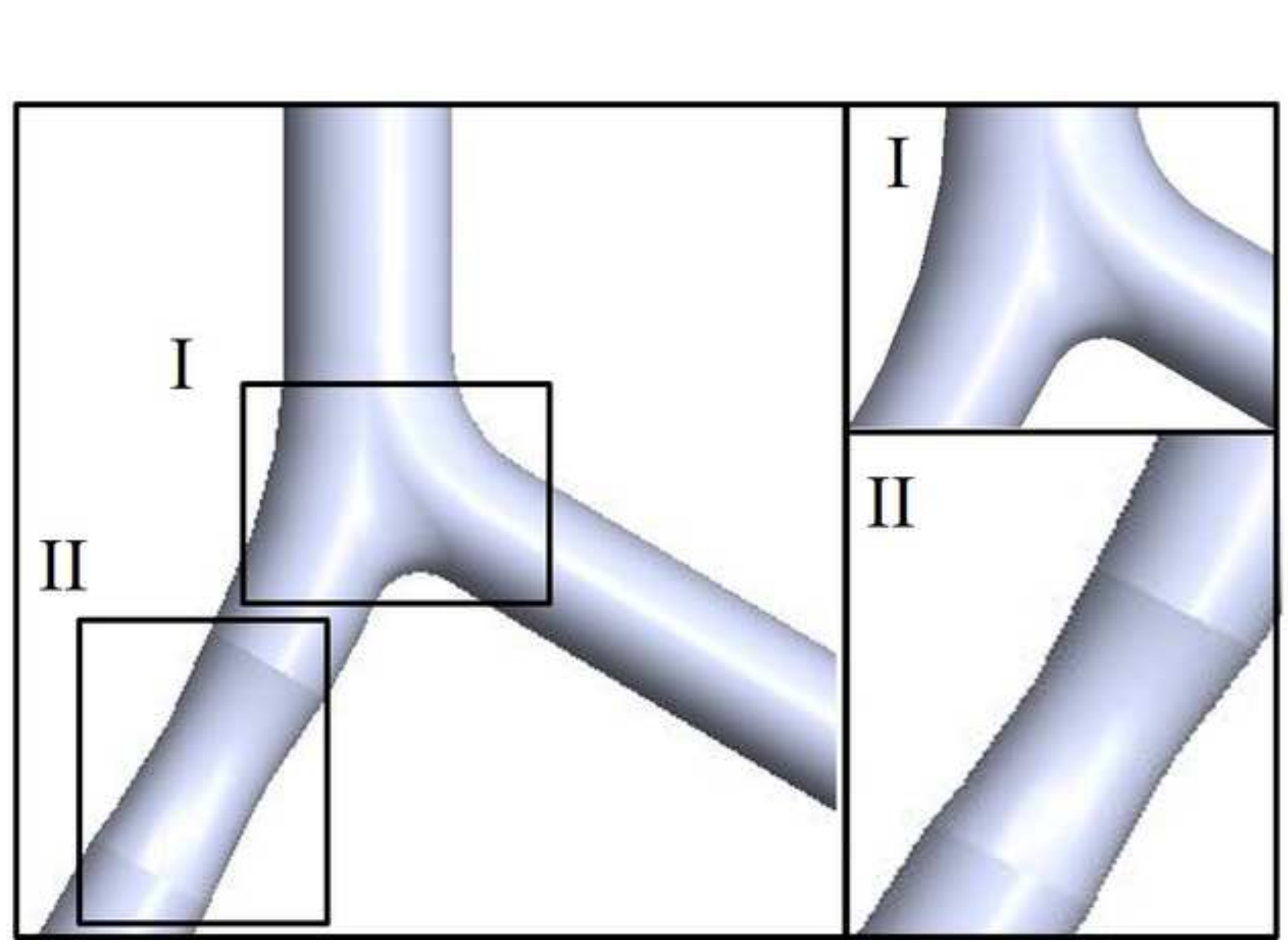




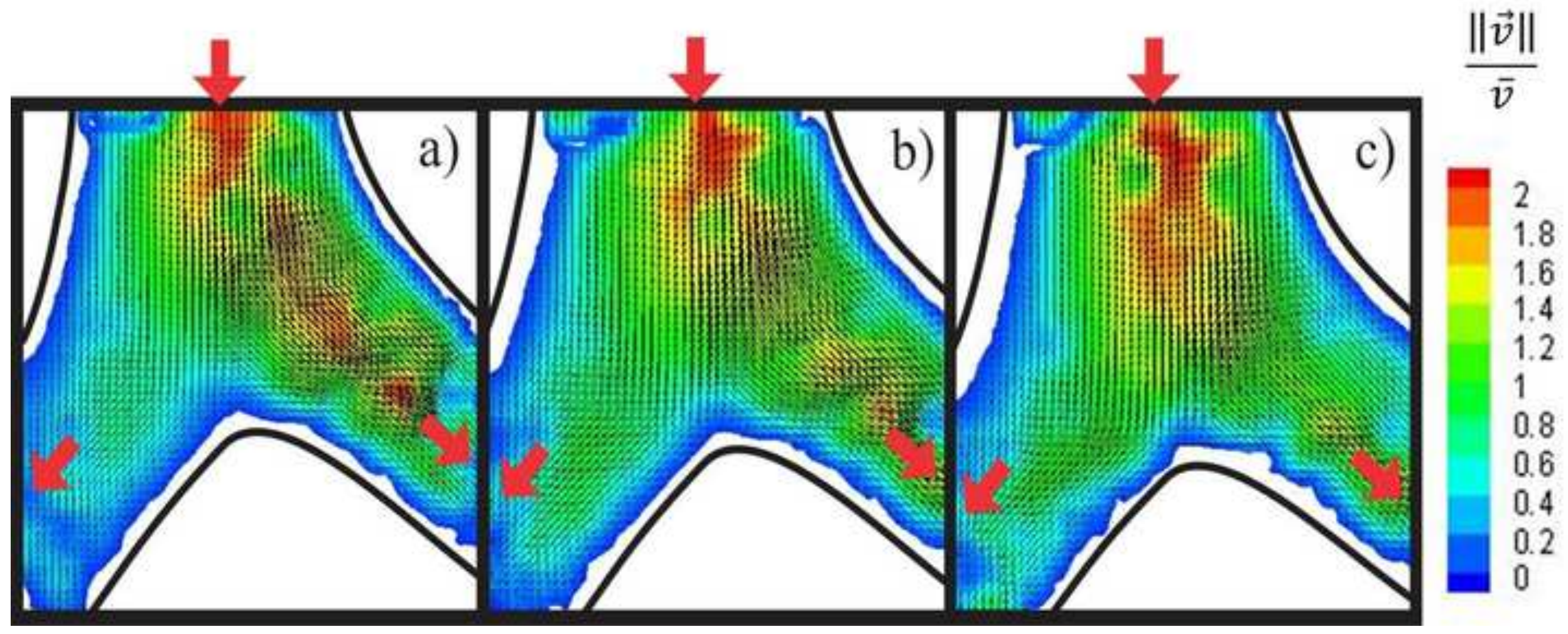




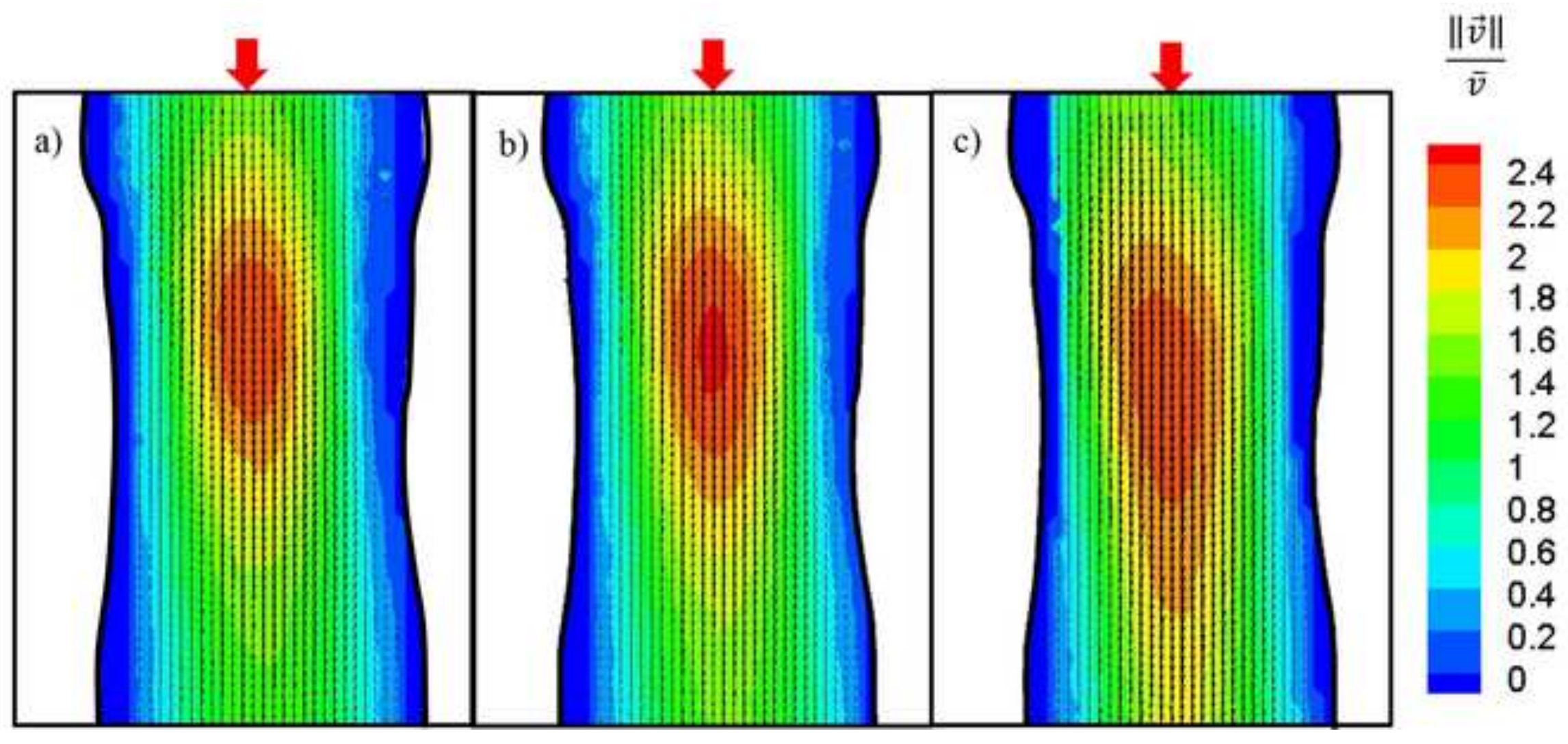




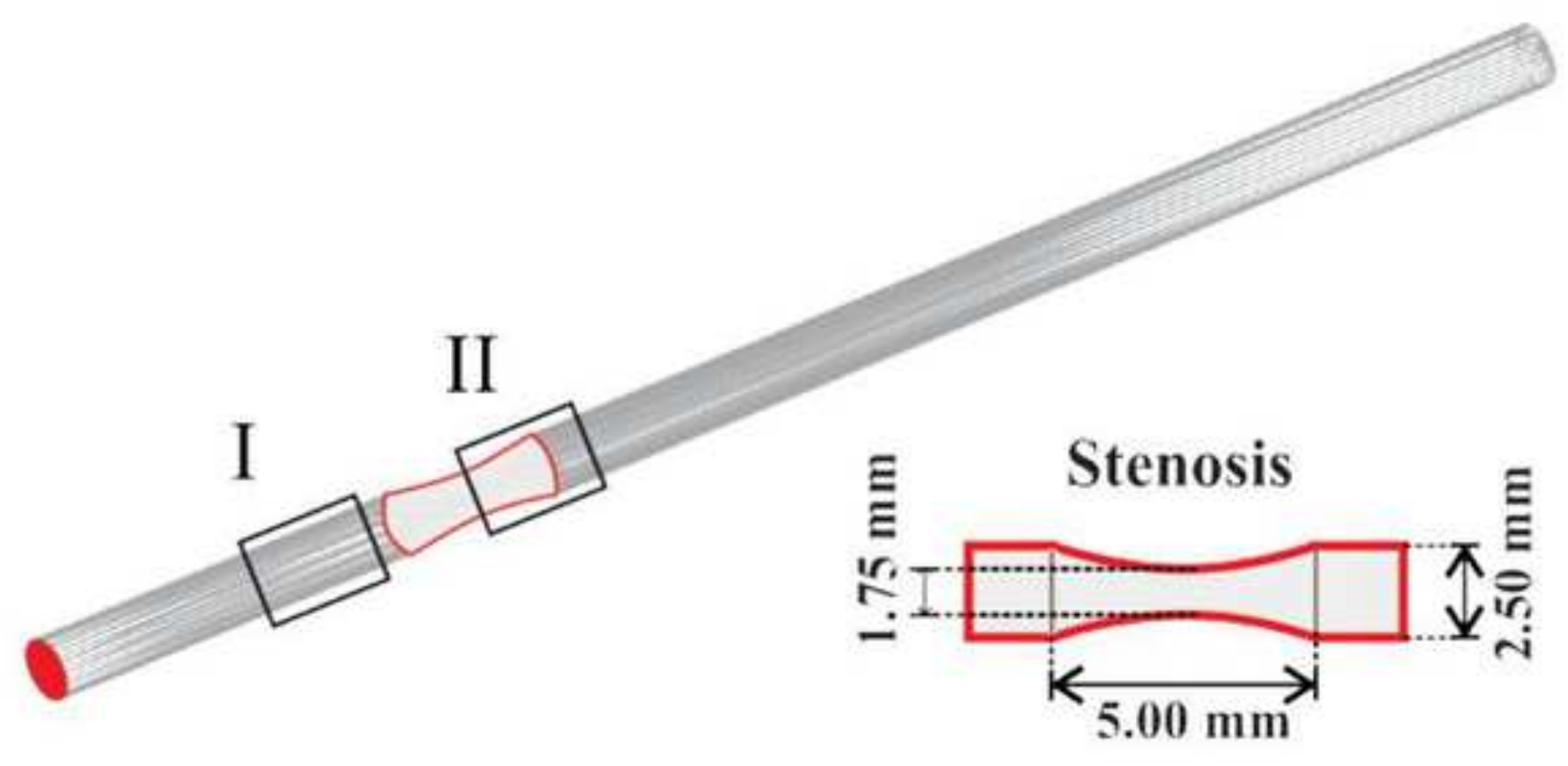



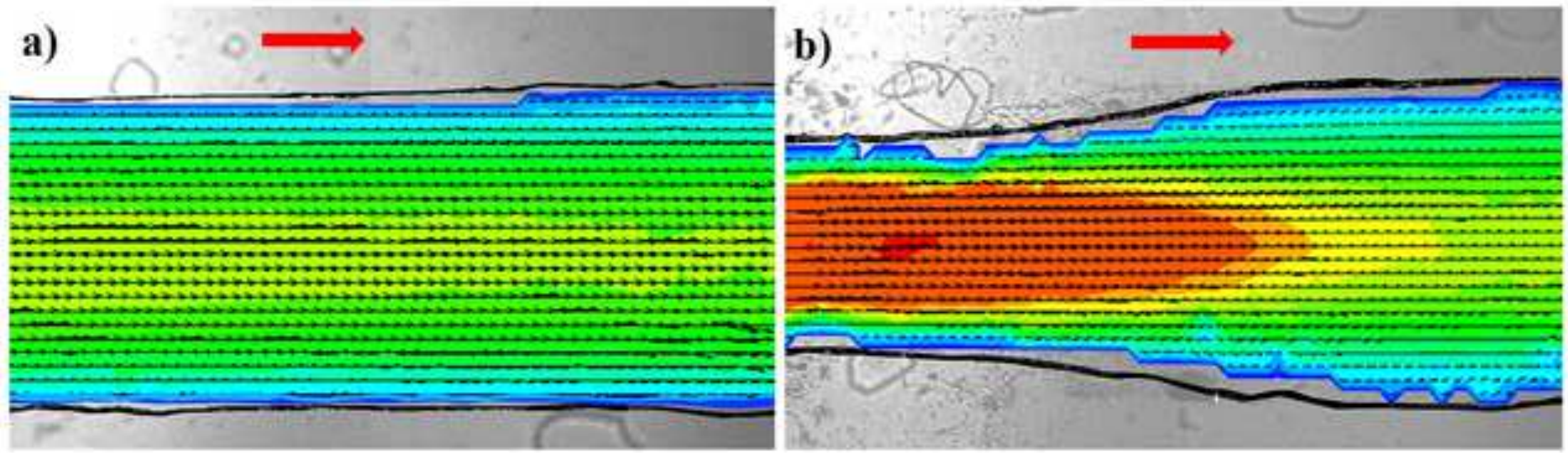

$\|\vec{v}\| m \cdot s^{-I}$

0.3

0.2

0.18

0.16

0.14

0.12

0.1

0.08

0.06

0.04

0.02

0.01

(1)




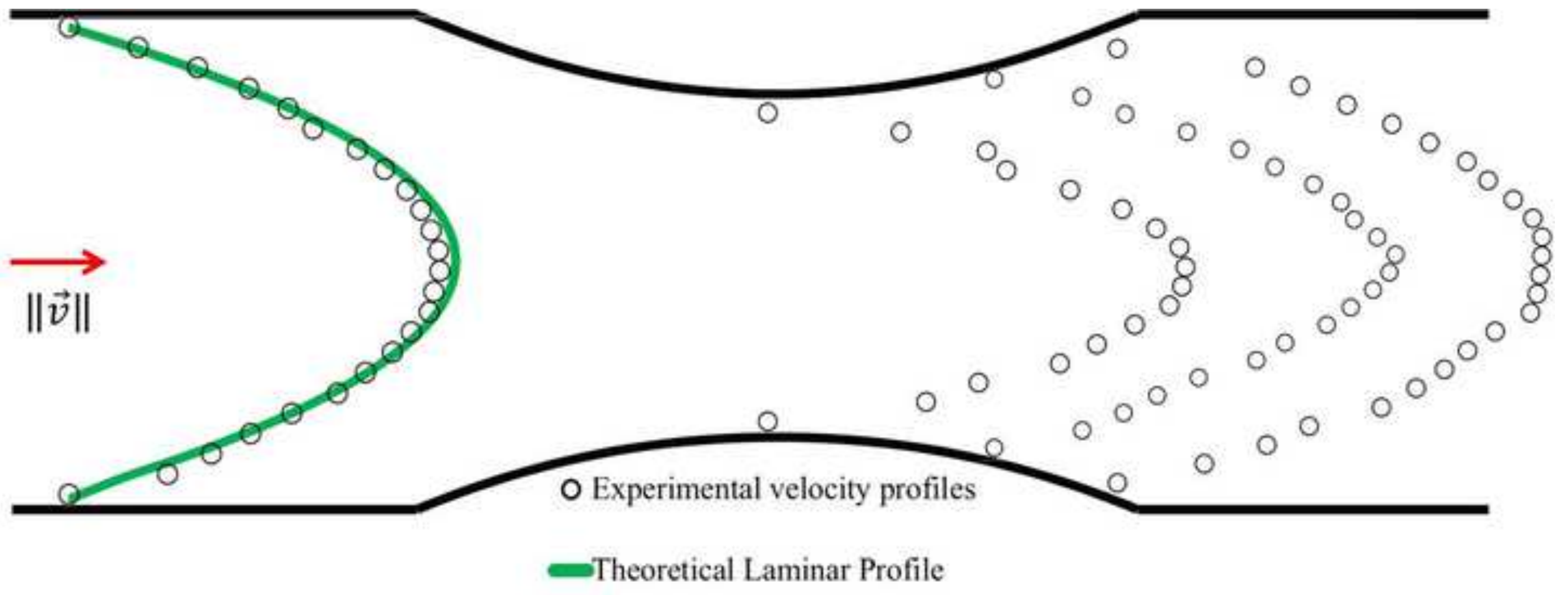


Tables

Table 1.

\begin{tabular}{cccc}
\hline Materials & Refractive index & $\begin{array}{c}\text { Density } \\
\mathbf{k g . m}\end{array}$ & $\begin{array}{c}\text { Viscosity } \\
\text { Pa·s }\end{array}$ \\
\hline Air & 1.0003 & 1.2041 & $1.837 \times 10^{-5}$ \\
Water & 1.3321 & 998.21 & $1.002 \times 10^{-3}$ \\
Water / glycerol & 1.4125 & 1156.6 & $1.140 \times 10^{-2}$ \\
PDMS & 1.4125 & - & - \\
\hline
\end{tabular}

Table 2.

\begin{tabular}{|c|c|c|c|c|c|c|c|c|}
\hline & & \multirow{3}{*}{$\begin{array}{c}\text { Straight } \\
\text { channel } \\
2.5\end{array}$} & \multirow{3}{*}{$\begin{array}{c}\text { Straight } \\
\text { channel } \\
3.5\end{array}$} & \multicolumn{5}{|c|}{ Bifurcated channel } \\
\hline & & & & \multirow[b]{2}{*}{ Inlet } & \multicolumn{2}{|c|}{ Outlet 1} & \multicolumn{2}{|c|}{ Outlet 2} \\
\hline & & & & & $\begin{array}{c}\text { Major } \\
\text { axis }\end{array}$ & $\begin{array}{c}\text { Minor } \\
\text { axis }\end{array}$ & $\begin{array}{c}\text { Major } \\
\text { axis }\end{array}$ & $\begin{array}{c}\text { Minor } \\
\text { axis }\end{array}$ \\
\hline CAD & $d / m m$ & 2.50 & 3.50 & 3.50 & 3.50 & 2.78 & 3.50 & 2.78 \\
\hline \multirow{2}{*}{ Paraffin } & $\bar{d} \pm \sigma / m m$ & $2.64 \pm 0.31$ & $3.67 \pm 0.07$ & $3.7 \pm 0.11$ & $3.69 \pm 0.19$ & $2.36 \pm 0.15$ & $3.45 \pm 0.16$ & $2.50 \pm 0.23$ \\
\hline & $\boldsymbol{\epsilon} / \%$ & 5.41 & 4.61 & 5.28 & 5.15 & 17.70 & 1.45 & 11.02 \\
\hline \multirow{2}{*}{ Sucrose } & $\bar{d} \pm \sigma / m m$ & $2.43 \pm 0.02$ & $3.49 \pm 0.07$ & $3.29 \pm 0.07$ & $3.31 \pm 0.04$ & $2.49 \pm 0.08$ & $3.42 \pm 0.10$ & $2.62 \pm 0.16$ \\
\hline & $\boldsymbol{\epsilon} / \%$ & 3.08 & 0.13 & 6.12 & 5.83 & 11.29 & 2.32 & 5.78 \\
\hline
\end{tabular}

